\title{
Computer Simulation of Irradiance Measurements from Aircraft
}

\author{
By \\ Michael R. Poellot and Stephen K. Cox
}

Department of Atmospheric Science

Colorado State University

Fort Collins, Colorado

This research was funded by the Office for Climate Dynamics of the National Science Foundation under Grant DES-7201681.

January 1975

\section{Department of Atmospheric Science}




\title{
COMPUTER SIMULATION OF \\ IRRADIANCE MEASUREMENTS FROM AIRCRAFT
}

\author{
by \\ Michael R. Poellot \\ and \\ Stephen K. Cox
}

This research has been funded by the Office for Climate Dynamics of the National Science Foundation under Grant DES-7201681.

\author{
Department of Atmospheric Science \\ Colorado State University \\ Fort Collins, Colorado \\ 80523
}

January 1975

Atmospheric Science Paper No. 233 


\section{ABSTRACT}

A computer simulation has been developed to optimize the use of the aircraft platform for the measurement of short wave irradiances. This model simulates the measurement of radiative fluxes in order to determine the approximate sample sizes required under various conditions of cloudiness.

The simulated required sampling length or averaging distance was found to be inversely proportional to the height of the sensor above or below the cloud field. The magnitude of the averaging distance and the rate of its decrease with height are the result of signal variations on two scales. Near the cloud surface, the data has a high variance due to small scale, large amplitude variations in the irradiance. These fluctuations are rapidly smoothed as the aircraft-cloud separation increases. The longer period oscillations are not as easily smoothed. Then the aircraft is farther from the cloud, the large scale effects become the primary control on the averaging distance.

Six specific tropical cloud types were analyzed and averaging distances computed. These results may be used in real time deicsions concerning aircraft sampling altitudes and distances and may also be utilized in the analys is of aircraft radiation data.

A special technique is also outlined for the analysis of data exhibiting a bi-modal distribution of radiant fluxes. For this special case, a representative mean irradiance may be determined with a relatively small amount of data. 


\section{ACKNOWLEDGEMENTS}

The authors would like to express their appreciation for the theoretical assistance supplied by Dr. Thomas Keefe and Dr. Thomas McKee. Special thanks are due to Ms. Charlene Polifka for her very capable programming assistance, to Ms. Susan Kueh1 for typing the manuscript, and to Ms. Karla Garretson for drafting the figures.

Acknowledgement is made to the National Center for Atmospheric Research, which is sponsored by the National Science Foundation, for the computing time used in this research. This research has been funded by the Office for Climate Dynamics of the National Science Foundation under Grant DES-7201681. 


\section{TABLE OF CONTENTS}

PAGE

ABSTRACT

ACKNOWLEDGEMENTS

TABLE OF CONTENTS

iv

LIST OF TABLES

LIST OF FIGURES

vi

LIST OF SYMBOLS

vii

I. INTRODUCTION

II. FLUX MEASUREMENT MODEL

A. Geometry of the Hemispheric Sensor 5

B. Model Sampling Goemetry 11

C. Cloud Field Representation 14

D. Analysis of the Simulated Data 16

III. RESULTS 21

IV. APPLICATIONS OF RESULTS FOR THE GATE 28

A. Averaging Distances for Six Cloud Types 28

B. A Bi-Modal Analysis Technique 33

C. Applications to Surface Measurements of Irradiance 34

V. CONCLUSIONS 36

REFERENCES 38

APPENDIX A: Averaging Distances for the GATE 39

APPENDIX B: Sample Simulated Cloud Arrays 46 


\section{LIST OF TABLES}

Table I. Comparison of Square and Circular f.o.v. Samples

Table II. Comparison of Averaging Distance and Standard Deviation

Table IIIa. Averaging Distances for Simulated Randomly Dispersed Cloud Elements and Resulting Cloud Fields

Table IIIb. Averaging Distances for Fixed Coordinate Cloud Fields 


\section{LIST OF FIGURES}

Figure 1. Comparison of hypothetical sinusoidal and uniform signals and their cumulative means.

Figure 2. Geometry of irradiance above an isotropic reflector.

Figure 3. Relative anisotropy of stratus clouds as a function of relative azimuth $(\psi)$ and radiometer zenith angle $(\theta)$, for solar elevation $=17-18^{\circ}$ and spectral regions $0.55-0.85 \mu$ and $0.2-4.0 \mu$. (from Salomonson and Marlatt, 1968)

Figure 4. Geometric weighting of sample value.

Figure 5. Cumulative mean of simulated irradiance time series and error interval $\mu+\varepsilon \mu$ for two different starting points in the same series.

Figure 6. High frequency variations (solid line) superimposed on low frequency variations (dashed line). Dashed line represents 50 sample averages.

Figure 7. Power spectrum analysis of large scale variations in simulated irradiance time series, over cumulus cloud areal coverages of $10 \%, 50 \%$, $90 \%$.

Figure 8. Simulated distribution of cumulus clouds.

Figure A1. Averaging distance for scattered cumulus as a function of cloud-aircraft separation and \% cloud cover.

Figure A2. Same as Figure AT, but for broken cloud deck.

Figure A3. Averaging distance for uniform cloud deck as a function of cloud-aircraft separation.

Figure A4. Same as Figure A3, but for closed cellular convection.

Figure A5. Same as Figure A3, but for street cumulus.

Figure A6. Same as Figure A3, but for cloud bands.

Figure B]. Plot of simulated cumulus cloud array, $20 \%$ areal coverage.

Figure B2. Plot of simulated broken cloud deck, $80 \%$ areal coverage.

Figure B3. Plot of simulated closed cellular convection.

Figure B4. Plot of simulated street cumulus.

Figure B5. Plot of simulated cloud bands. 


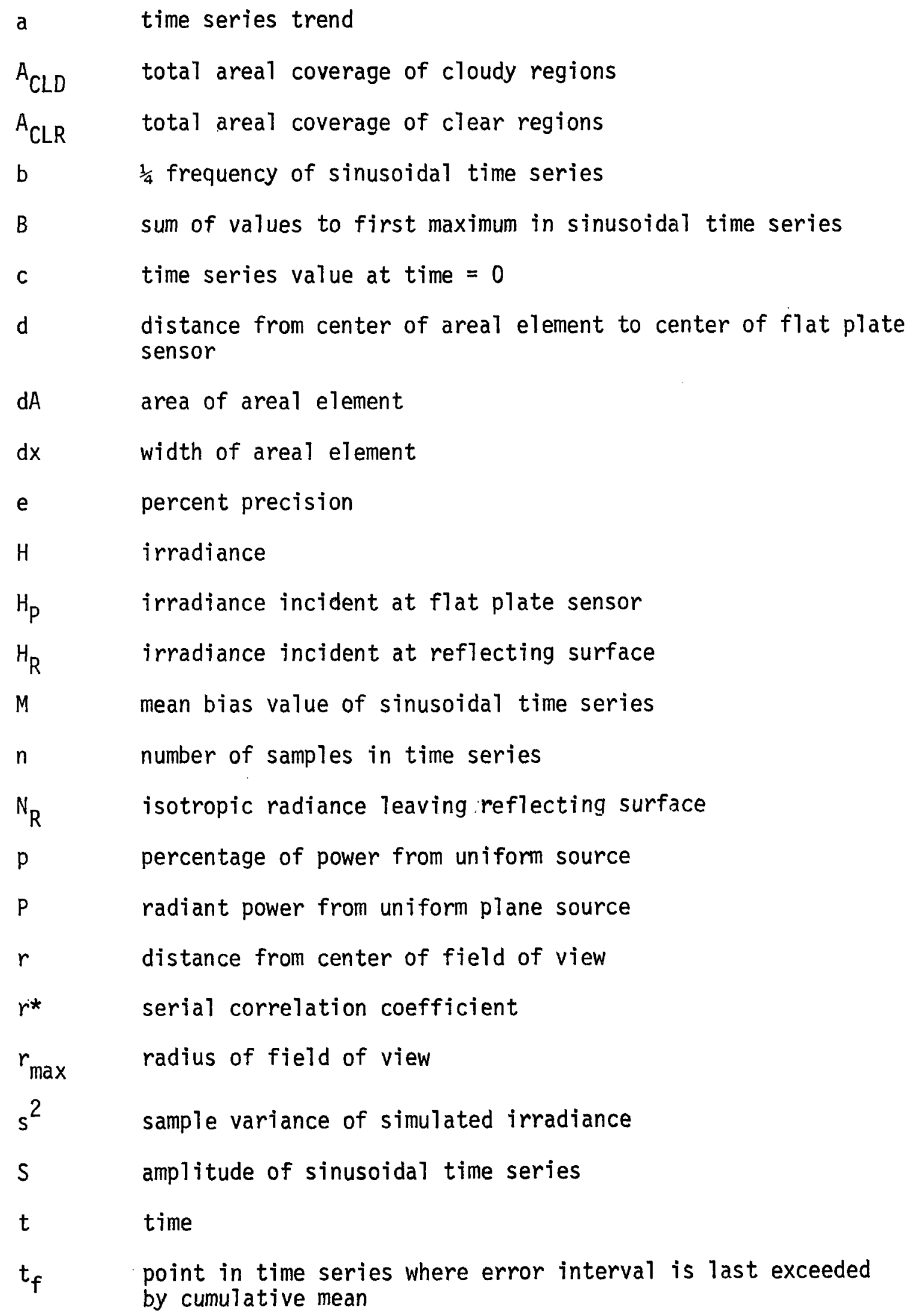




\section{LIST OF SYMBOLS - Continued}

\begin{tabular}{|c|c|}
\hline$W(r)$ & geometric weighting function \\
\hline$W T(t)$ & time response weighting function \\
\hline$x$ & width of square field of view \\
\hline$x$ & time series of simulated irradiance samples \\
\hline$\vec{x}$ & area weighted mean of irradiance time series \\
\hline $\bar{x}_{C L D}$ & time series mean irradiance over cloudy areas \\
\hline $\bar{x}_{\text {CLR }}$ & time series mean irradiance over clear areas \\
\hline$x_{i}$ & term of time series of simulated irradiances \\
\hline $\bar{x}_{t}$ & cumulative mean of time series of simulated irradiances \\
\hline$x_{\text {TV }}$ & clear-cloud threshold value of irradiance \\
\hline$y(t)$ & element of sinusoidal time series \\
\hline $\bar{y}_{\mathrm{t}}$ & cumulative mean of sinusoidal time series \\
\hline$z$ & $\begin{array}{l}\text { normal component of distance from sensor to plane of cloud } \\
\text { surface }\end{array}$ \\
\hline$\varepsilon$ & $\frac{1}{2}$ width of error interval about mean \\
\hline$\theta$ & zenith angle, nadir angle \\
\hline$\theta^{\prime}$ & truncation angle for finite field of view \\
\hline$\lambda$ & T/e time constant \\
\hline$\mu$ & true mean of sampled irradiance population \\
\hline p & reflectivity \\
\hline$\rho_{\mathrm{d} A r}$ & $\begin{array}{l}\text { reflectivity of areal element at a distance } r \text { from center of } \\
\text { field of view }\end{array}$ \\
\hline$\sigma$ & standard deviation of sampled irradiance population \\
\hline$\tau$ & coefficient of variation \\
\hline$\phi$ & azimuth angle \\
\hline$\psi$ & relative azimuth angle \\
\hline (w) & solid angle \\
\hline
\end{tabular}




\section{INTRODUCTION}

The GARP Atlantic Tropical Experiment (GATE) Radiation Subprogram (RSP) (Kraus et a], 1973) has as a primary objective the determination of the vertical profiles of radiative flux divergence in the tropical atmosphere. While flux divergence measurements may be made from upper air balloons, (Suomi and Kuhn, 1958), the airplane provides the most versatile platform for accurate radiative flux measurements. Radiometersondes yield only one sample of the net flux at each level in the vertical, and these profiles are limited in space to the vicinity of the launching site. The aircraft platform makes it possible to measure flux divergence over large prespecified volumes of the atmosphere. The numerous samples of the net flux collected at each level also reduce the RMS error of the divergence.

Since the allocation of dedicated aircraft time (or sorties) to the RSP was 1 imited, the optimal use of this time was essential. Part of this optimization required an efficient, systematic collection of data. For a given set of parameters both over- and under-sampling waste resources. Heterogeneous conditions of cloudiness present special problems. Consider the time series of sample values of radiant flux in Figure 1a. Partly cloudy conditions are represented by the sinusoidal curve and a horizontally uniform field is shown by the dashed line. If the mean flux is determined by the convergence of the cumulative mean (Fig. Ib.), the mean of the uniform field is given by the initial value. However, many samples are needed to determine the mean under heterogeneous conditions. It is the purpose of the computer simulation described in this report to provide a set of sampling time guidelines for operational use in the field and in subsequent data reduction. 

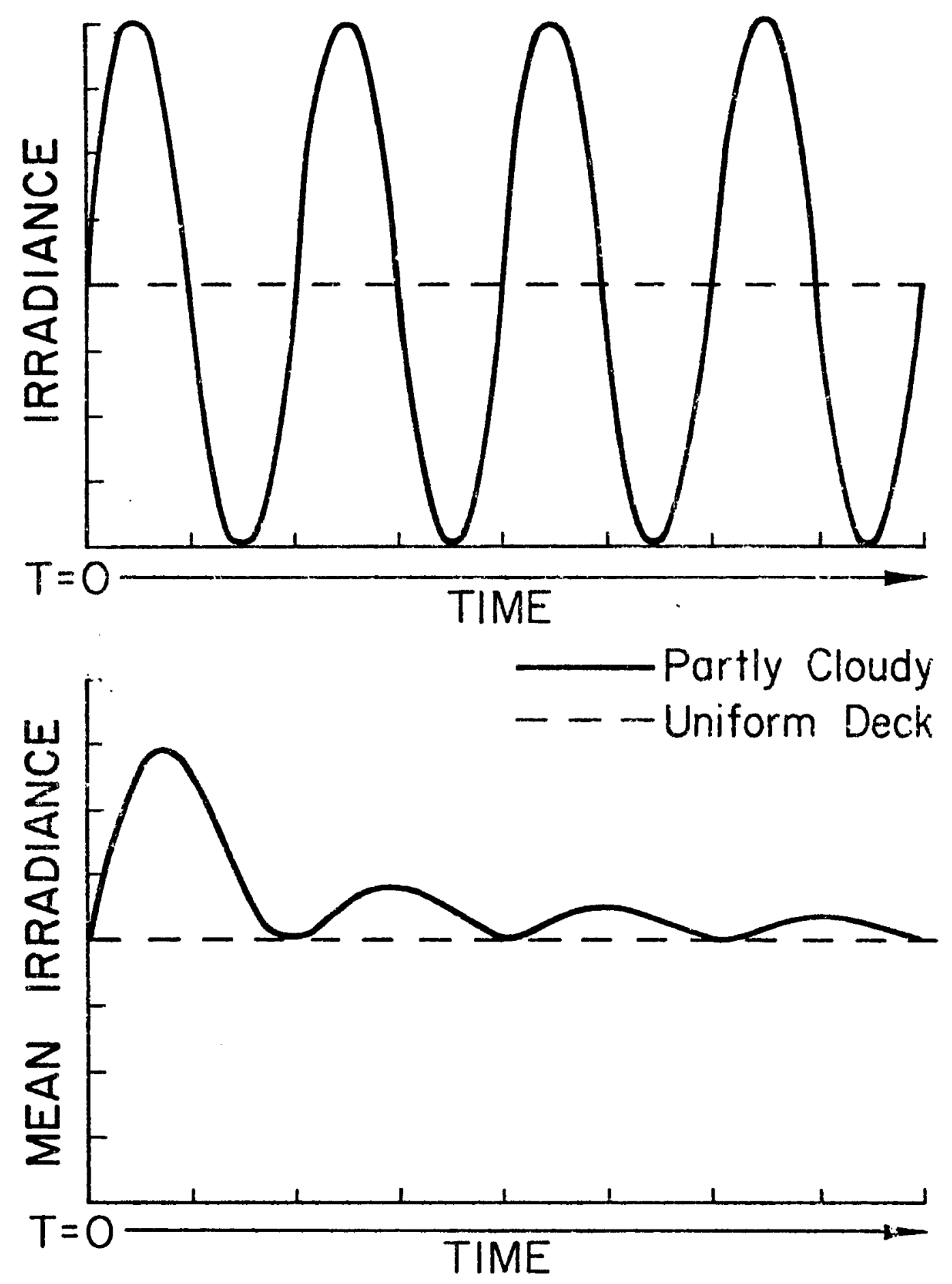

Figure 1. Comparison of hypothetical sinusoidal and uniform signals and their cumulative means. 
In middle latitude disturbances, where horizontal temperature and moisture gradients are large, the radiative component of the net energy budget is not significant on the synoptic scale. However, in the quasisteady state regime of the tropical atmosphere, the radiative heating plays an important role. Reed and Recker (1971) reported the net radiative temperature change in a tropical wave disturbance to be of the same order of magnitude as the total diabatic heating. Yanai, et al (1973) have shown that the radiative heating is a significant component of the energetics of cloud clusters. Thus, the RSP has stated the desired resolution of radiative cooling rates as $\pm 0.2^{\circ} \mathrm{C}$ day $^{-1}$ for a six to twelve hour period over a $200 \mathrm{mb}$ thick layer of the atmosphere. This implies individual flux measurements or mean values must have a relative accuracy of $\pm 2.4 \mathrm{wm}^{-2}$ per $200 \mathrm{mb}$. To achieve this degree of accuracy under heterogeneous conditions, it is felt that a realistic radiation budget must be obtained through bulk measurements and statictical techniques (Cox and Vonder Haar, 1973). The approach developed by Cox (1973) will be related here to the problems of airborne flux measurements.

The composite technique of Cox is based on the assumption that similar cloud types and patterns have similar radiative properties. Each configuration thus has a characteristic radiative cooling profile. If enough measurements of the flux profiles are made for various tropical cloud formations, a mean cooling profile may be determined for each type. These values may than be applied to a large volume of the atmosphere through a knowledge of the cloud population.

In the GATE, these measurements were made with hemispheric $(2 \pi$ steradian) radiometers. Sampling runs were made by one or more aircraft at several levels relative to a selected cloud feature, both above and 
below the clouds. The mean irradiances calculated from the data collected at these levels give a divergence profile for that cloud type.

The problem which remains is to determine how large of a sampling volume is needed to obtain statistically significant mean irradiance values. There are many factors which affect the relative accuracy of the mean values derived from the aircraft data. These include random and systematic errors in the instruments and data recording systems, departure from perfect cosine response of the instrument, deviations from level flight, changes in solar geometry, and the natural variability of the irradiance impinging on the sensors. The largest uncertainty and the factor being considered here is the signal variability in the presence of heterogeneous partly cloudy conditions. In this case, the short wave $(.3 \mu-3 \mu)$ fluxes often vary by a factor of four or more. This may decrease the precision of a measured statistical mean representative of a large population, and hence decrease the relative accuracy of the mean fluxes used to calculate the flux divergence. Since the infrared $(3 \mu-50 \mu)$ fluxes are considerably less variable, this study approaches the problems of determining mean short wave irradiances from the aircraft platform. 


\section{FLUX MEASUREMENT MODEL}

The need for a computer simulation of irradiance measurements from an aircraft platform is real. The present resources of this type of actual data are simply inadequate to deduce a set of optimal sampling times. Sampling legs flown in previous field experiments were often of short duration or not accompanied by sufficient verbal or visual descriptions. Data for some cloud types is practically nonexistant. The model described in this report compensates for the deficiency by generating a large data set for various specified cloud configurations, using characteristic cloud properties such as areal coverage and reflectivity as input. It is a three-part model which includes the storing of a simulated cloud field, the simulated sampling of this field, and the analysis of the simulated data.

\section{A. Geometry of the Hemispheric Sensor}

The basis of this model is the sampling of finite areal elements through the application of some simple radiation geometry. The hemispheric radiometers used in the GATE have a flat plate sensor surface. Using the reflection of solar radiation from an isotropic plane surface $R$ as an example, the geometry of the radiant flux is shown in Figure 2 . The flat plate, $P$, is plane parallel to $R$.

The radiance $N_{R}$ reflected from the isotropic surface is assumed to be independent of $\theta$ and is related to the irradiance $H_{R}$ incident at the surface by

$$
N_{R}=\frac{\rho H_{R}}{\pi},
$$

where $\rho$ is the reflectivity of the surface. The irradiarce passing through a surface perpendicular to $N_{R}$ is defined as 


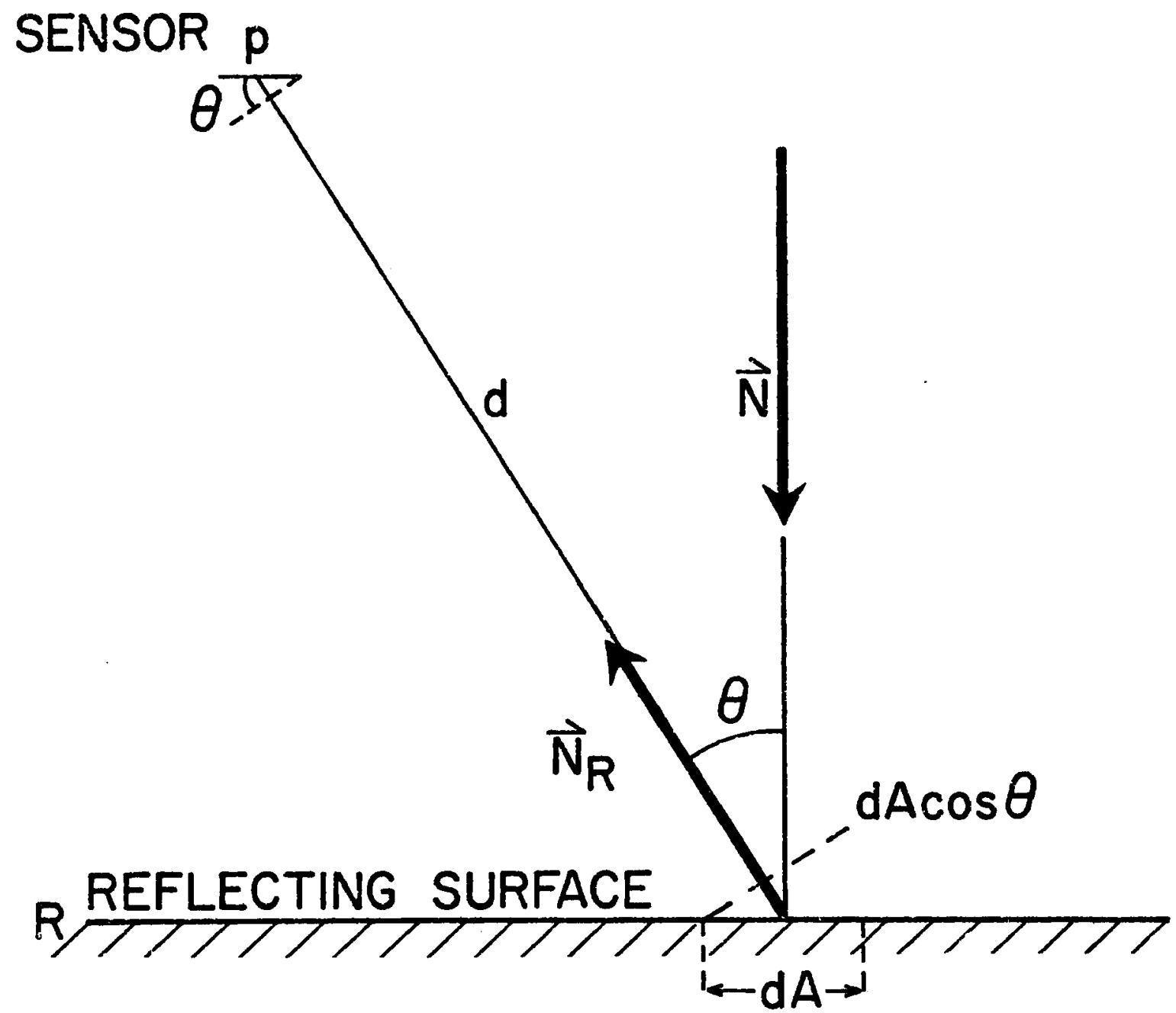

Figure 2. Geometry of irradiance above an isotropic reflector. 


$$
H=\int_{0}^{\omega} N_{R} d \omega,
$$

where $w$ is the solid angle of a cone about $N_{R}$. The contribution to the irradiance at $P$ from an areal element of the surface $d A$ is then

$$
\Delta H_{P}=N_{R} \cos \theta \Delta \omega \text {. }
$$

The factor $\cos \theta$ is introduced to account for the effect of the angle $\theta$ between $P$ and $N_{R}$. The solid angle subtended by $d A$ at a distance $d$ from $P$ is

$$
\Delta \omega=\frac{d A \cos \theta}{d^{2}} .
$$

Substituting in Eq. (3) for $N \Delta w$ from Eqs. (1) and (4) yields

$$
\Delta H_{P}=\frac{\rho H_{R} d A \cos ^{2} \theta}{\pi d^{2}} .
$$

The total irradiance at $P$ is the integral sum of $\Delta H_{p}$ for all $d A$ from $\theta=0$ to $\theta=\pi / 2$ over all azimuth angles $\phi=0$ to $\phi=2 \pi$. This expression may be used to calculate the irradiance at any point as the sum of the contributions from a plane array of finite areal elements, such as a cloud field. For a point above cloud top, reflected short-wave radiance is considered and the elements are assigned relative values of reflectivity. Between-cloud elements are given a value for the underlying surface and atmosphere. From below cloud level, transmitted radiances are represented by relative transmissivities for the cloud and clear sky areas. The discussion in the remainder of section II will refer only to the abovecloud case.

One of two assumptions made here is that all of the areal elements lie in the same plane. This is reasonable for the cloud types being 
studied, particularly when the sensor is considered to be on the order of 1000 feet from cloud top.

It is also assumed that the anisotropic effects of the clouds are negligible, provided that the measurements are made at low solar zenith angles. Salomonson and Marlatt (1968) presented the anisotropy of stratus clouds in terms of relative anisotropy. This is a measure of the error in the albedo which would result if anisotropic effects are ignored. For a solar zenith angle of $17^{\circ}-18^{\circ}$, the relative anisotropy as a function of radiometer nadir angle $\theta$ and relative azimuth is shown in Figure 3 .

As viewed from an aircraft flying over a uniform cloud deck, the anisotropy would appear as a bright spot off to one side. In this instance, the anisotropic effect would bias the mean value of the reflected radiation, but the convergence of the cumulative mean and hence the optimal sample size would remain virtually unaffected.

Over partly cloudy conditions, anisotropic effects would be greatest in two separate cases. One is if the maximum of anisotropy is centered over a cloud while the aircraft is also over a cloud. Ignoring the anisotropy here would cause an underestimate of the peak values of the sample time series to be made. The second case would find the aircraft centered over a clear area while the "bright spot" again covers a cloud. An assumption of isotropy here would lead to an underestimate of the time series minima. If these two cases are equally likely to occur, the effect of the anisotropy again is to bias the mean, but leave the convergence time virtually unaffected. Thus, for small solar zenith angles, the assumption of isotropy for our purposes is valid.

These anisotropic effects could be included in the model if desired for a different application. Empirical observations like those 
$-9-$
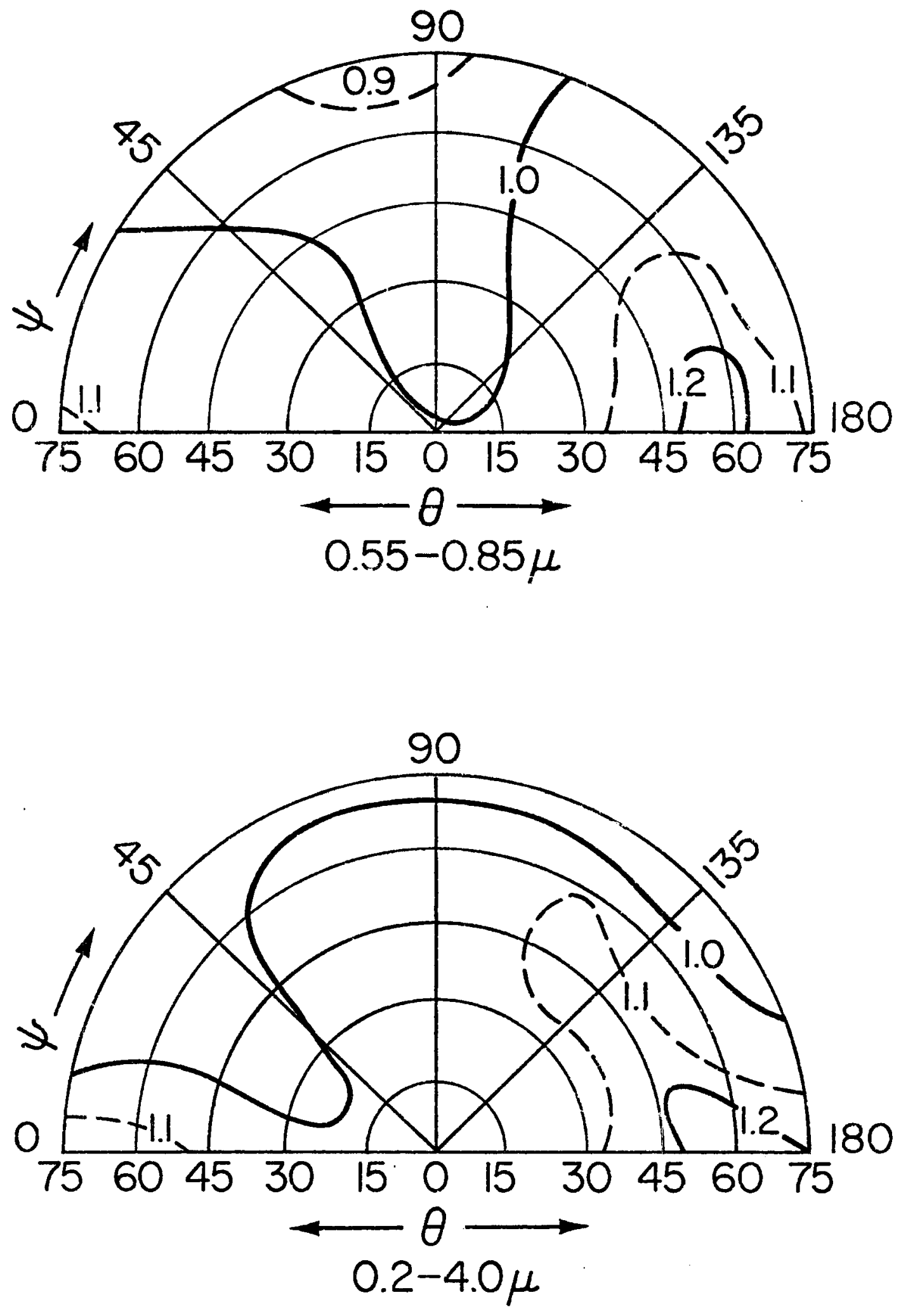

Figure 3. Relative anisotropy of stratus clouds as a function of relative azimuth $(\psi)$ and radiometer zenith angle $(\theta)$, for solar elevation $=17-18^{\circ}$ and spectral regions $0.55-0.85 \mu$ and $0.2-4.0 \mu$. (from Salomonson and 
of Salomonson and Marlatt may be developed into a table or matrix of anisotropic factors (Sikula and Vonder Haar, 1972). This matrix would then be applied to the simulated cloud field to adjust the reflectivity values for the anisotropy.

An approximation must also be made to narrow the field of view (f.o.v.) of the sensor. The projection of a hemispheric f.o.v. on a plane surface is a circle with an infinite diameter. This diameter must be reduced to a useable size. This requires that the $2 \pi$ steradian f.o.v. be truncated at some nadir angle less than $90^{\circ}$. The radiant power from an infinite plane uniform source passing through a hemisphere of unit radius at an angle $\theta$ is proportional to $\sin \theta \cos \theta$. The total power contributed at all angles from 0 to $\theta^{\prime}$ is proportional to

$$
P \sim \int_{0}^{\theta} \sin \theta \cos \theta d \theta \text {. }
$$

For a $2 \pi$ steradian f.o.v., $\theta^{\prime}=\pi / 2$.

An f.o.v. which would encompass a given percentage, $p$, of the power from a uniform source would be defined by solving the following equation for $\theta^{\prime}$ :

$$
p=\frac{\int_{0}^{\theta^{\prime}} \sin \theta \cos \theta d \theta}{\int_{0}^{\pi / 2} \sin \theta \cos \theta d \theta} .
$$

Initially, the $99 \%$ power f.o.v. of $\theta^{\prime}=84^{\circ} 15^{\prime}$ was arbitrarily selected as the cutoff point. This was later reduced in all calculations for this paper to $\theta^{\prime}=83^{\circ} 10^{\prime}$ or $p=98.6 \%$. This reduction led to a decrease of $25 \%$ in the computer run time of the model without significantly affecting the integral sum of the elements. 


\section{B. Model Sampling Geometry}

In the simulation, the cloud fields are stored in a two-dimensional array. To maximize use of the core space within the computer, the areal elements are assigned relative reflectivities of integer values between 0 and 7. Each number is stored in a binary format which uses only three bits of a 60 bit word. This facilitates the storage of an array of integer numbers 20 times the size of a corresponding set of real values. While some flexibility and resolution is lost through the use of integer values, this is we 11 compensated for by the increase in the possible size of the stored cloud field.

The sampling of the cloud array is begun by centering the sensor over one of the areal elements at a simulated vertical distance $z$ from the plane of the clouds. All elements whose center coordinates lie within the f.o.v. are then included in the sample. The radius $r_{\max }$ of the f.o.v. is a function of $z$ and the limiting angle $\theta^{\prime}$. Using $\theta^{\prime}=83^{\circ} 10^{\prime}$ and referring to the Figure 4 , we see that

$$
r_{\max }=d \sin \theta^{\prime}=\frac{z \sin \theta^{\prime}}{\cos \theta}=8.35 \mathrm{z}
$$

Recalling Eq. (5), and letting $H_{R}=1$, the contribution of each element to the irradiance at point $P_{Z}$ is given by:

$$
H_{P}=\frac{\rho_{d A r} d A \cos ^{2} \theta}{\pi d^{2}},
$$

where

$$
\theta=\tan ^{-1}\left(\frac{r}{z}\right) \text {. }
$$

and $\rho_{\mathrm{dAr}}$ is the reflectivity assigned to the element $\mathrm{d} A$.

The relative weight of each element is a function of $i$ ts distance $r$ from the center of the f.o.v. For a fixed $z$, these weights need only be calculated once and then stored in a one-dimensional weighting array $W(r)$, defined as 


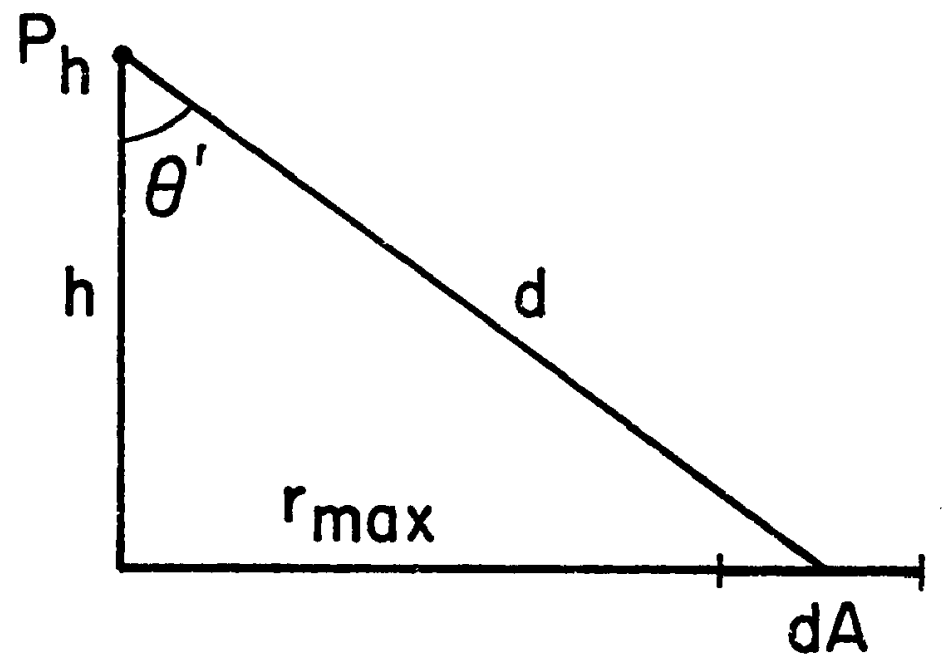

Figure 4. Geometric weighting of sample value. 


$$
\begin{aligned}
w(1) & =f(r)=\frac{d A \cos ^{2} \theta_{r}}{\vdots} \\
w\left(r_{\max }\right) & =f\left(r_{\max }\right)=\frac{\pi_{r}^{2}}{\pi d_{r_{\max }}^{2}} .
\end{aligned}
$$

The total irradiance incident at the sensor for an individual sample is then:

$$
H_{p} \cong \sum_{r=0}^{r=r} \max \rho_{d A_{r}} * W(r)
$$

The size of $W(r)$ is proportional to $z$. Subsequent samples are calculated by shifting the center point of the f.o.v. and then applying $W(r)$ to a new set of reflectivities. The propogation of the f.o.v. simulates aircraft motion.

The rate of this movement may be translated into real distances, sampling rates and aircraft speeds. For example, if $\mathrm{dA}$ is $100 \mathrm{~m}$ on a side $d x$ and $W(r)$ is shifted by $d x$ for each sample, this is equivalent to a sampling rate of $1 \mathrm{sec}^{-1}$ on an aircraft moving at $100 \mathrm{~m} \mathrm{sec}^{-1}$ relative to the clouds. These values may be adjusted to the desired scale and resolution.

For computational simplicity, it is desirable to approximate the circular f.o.v. by a square of width $2 r_{\max }$. The validity of this approximation is dependent on the number of elements integrated into each sample, which in turn is a function of $z$. Thus, the sample sums over a square and over a circle should converge as $z$ is increased. A "worst possible case" test of this hypothesis was performed. A circular array of values of radius $x / 2$ was stored within a square of width $x$. All elements within a distance $x / 2$ of the center were assigned a value of one while the rest were given a value of seven. The results 
of the test, shown in Table I, indicate that this approximation is good if $z \geq 3 \mathrm{dx}$, where $\mathrm{dx}$ is the element width. This is an integration of $57^{2}$ or 2601 elements for one sample.

Table I. Comparison of Square and Circular f.o.v. Samples.

\begin{tabular}{cccc}
$\mathrm{Z}$ & $\mathrm{H}$ square & $\mathrm{H}$ circle & $\mathrm{H}_{\mathrm{S}} / \mathrm{H}_{\mathrm{c}}$ \\
\hline 1 & 1.038 & 1.015 & 1.02 \\
2 & 1.830 & .989 & 1.85 \\
3 & 1.078 & .989 & 1.09 \\
4 & 1.036 & .990 & 1.05 \\
5 & 1.025 & .990 & 1.04 \\
\hline
\end{tabular}

The framework has thus been set up for sampling a theoretical cloud field through the application of a geometric weighting array to an array of numbers representing reflected irradiances from cloud elements. The simulated height of the sensor above the cloud field is changed by adjusting the size of the f.o.v. and recalculating the weighting array. The width of the stored field is fixed by the f.o.v. while the length varies with the type of cloud being stored.

\section{Cloud Field Representation}

Two methods of storing cloud fields were devised to simulate the cloud types being studied. The first utilizes a direct input of the simulated cloud segment coordinates and their reflectance values. Cloud types processed for the GATE using this method include organized "street" cumulus and cloud bands. The second method uses an internal random routine of generating and storing the positions and reflectivity values 
of the segments within given guidelines of their characterisiic properties. Tropical cloud types studied in the manner were unorganized trade cumulus, uniform cloud decks, broken decks, and stratocumulus "closed cellular convection".

The simplest cloud form dealt with was cloud bands stored directly from input of punched cards. Each card contained the $x, y$ coordinate boundaries of a cloud band stored in the array. These clouds were assumed to be of uniform brightness and width and of infinite length, equally spaced, and oriented perpendicular to the line of flight of the aircraft. The bands were stored as rectangles extending across the width of the array. Because of their repetitive nature, only a limited number of bands had to be stored and sampled. The resultant data series could then be iterated to produce a sample of any length.

The other cloud type treated in this way was the case of trade cumulus which are organized into rows or "streets". These clouds were stored in rows as distinct square of uniform brightness, spacing and size. clouds in adjacent rows need not have the same characteristics. The sensor was simulated to pass perpendicular or parallel to the rows, over or between the clouds. Again, only a small number of rows were stored because the data array could be traversed as many times as desired.

The random technique of storing clouds was developed for two reasons. First, the unorganized nature of the cloud fields which it simulates requires the storage of very large arrays. These data cannot be iterated from a small set, and the time involved in storing cloud segments by fixed coordinates is prohibitive. Secondly, this random routine eliminates any bias which might be introduced by selecting cloud positions through the first storing method. As viewed from a satellite or ground platform, the spacing and thickness of the clouds at times does appear to be random. 
The essence of this technique is to allow three parameters to vary randomly within prespecified ranges. These are: the dimensions of the simulated cloud or clear rectangles, the brightness value assigned to the rectangle, and the ratio of cloud to clear areas. The box dimensions and values may be weighted to any desired distribution. These parameters are randomized by initializing and calling a random number generator in the computer system. The basic random technique was used to simulate the broken cloud decks. Variations of this for the other cloud types will be discussed with the model applications for GATE.

\section{Analysis of the Simulated Data}

Once the cloud field has been stored, the weighting array is centered over one end of the rectangle; the weighting array is then propogated one element at a time along the field length until it has traversed the entire box. At each point, the array is sampled and the result is a time series of relative irradiances $x_{i}$ :

$$
x_{i}=x_{1}, x_{2}, \ldots x_{n},
$$

where $n$ equals the field length minus the width of the f.o.v. This is the raw data set which is to be analyzed to determine the optimal averaging distance.

The averaging distance (a.d.) is defined by the number of samples required to yield a mean value of a cloud field that is precise to within e\% at an approximate $99 \%$ level of confidence. This number is converted into a distance by scaling to the desired resolution of the array. The $\mathrm{e} \%$ precision is attained by first calculating the mean value of the time series. The true mean $\mu$ of the cloud field is assumed equal to the sample mean. A cumulative mean is also calculated using the following expression: 


$$
\bar{x}_{t}=\frac{\sum_{i-1}^{t} x_{i}}{t},
$$

where $\bar{x}_{t}$ is the cumulative mean of the time series from the initial point to time $t$. Although $\bar{x}_{t}$ may oscillate about $\mu$, in time it converges to $\mu$. An error interval about $\mu$ is defined by $\mu \pm \varepsilon$ where $\varepsilon=e_{\mu}$. The point in the time series at which the cumulative mean falls and stays within the error interval gives the required sample size. An example is shown in Figure 5.

The approximate $99 \%$ level of confidence is a constraint which is applied to the averaging distance to insure that the sample size will be sufficient for $99 \%$ of 211 the cases expected. To observe an averaging distance which would cover all possible cases would be impractical .

For a given cloud field, different averaging distances may result if the cumulative mean is started at different points in the time series. This is due to the strong influence on the characteristics of $\bar{x}_{t}$ by the initial values of the sum. Curves representing $\bar{x}_{t}$ for two different starting points in the same series are shown in Figure $5 \mathrm{a}$ and $5 \mathrm{~b}$.

The cumulative mean may be calculated from various initial points if the series is made continuous by setting $x_{n+i}=x_{i}$. To avoid a discontinuity between $x_{n}$ and $x_{n+1}$, the cloud field is extended before sampling by reproducing at the end of the array the elements of the first sample square. As these values are included in the samples, the final values of $x_{i}$ are forced to converge with those at the beginning, or $x_{\mathrm{n}} \cong x_{1}$.

If $\bar{x}_{t}$ is calculated for 100 distinct starting points distributed throughout the time series, the approximate $99 \%$ level of confidence is 

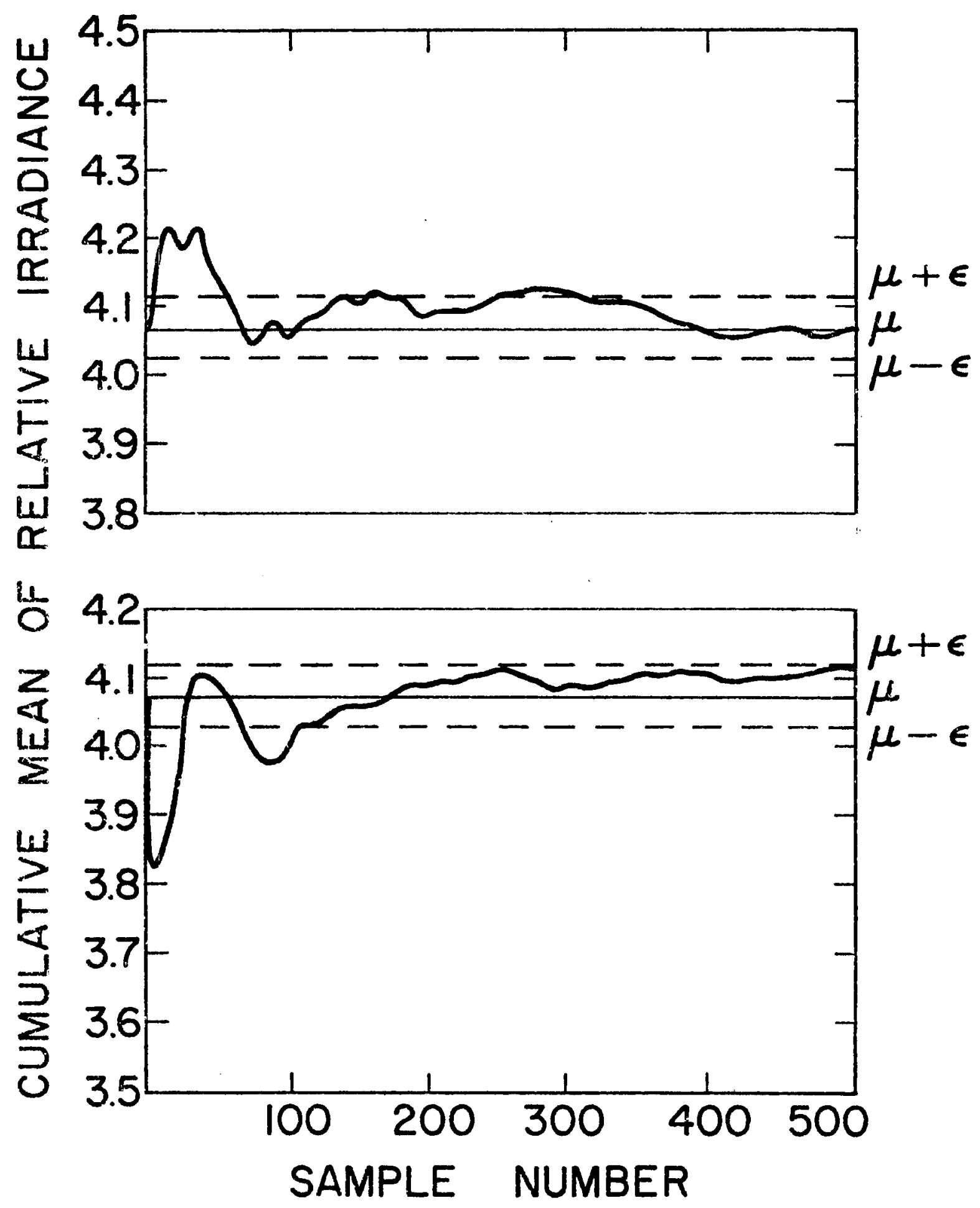

Figure 5. Cumulative mean of simulated irradiance time series and error interval $\mu \pm \varepsilon \mu$ for two different starting points in the same series. 
represented by the second largest averaging distance. This value, however, may vary in the random cloud case for different arrays having the same characteristics. To maintain the high level of confidence, a number of similar cloud fields must be generated and sampled by changing the initial random number in the program. Ideally, 50 or 100 different fields should be analyzed for each case, but computer time restraints normally limit this number to ten or less. The approximate $99 \%$ level is held by selecting the largest a.d. of these cases.

Two operations are performed on the time series before it is analyzed to the desired accuracy. First, a weighting function is applied to introduce the time lag of the instrument. The $1 / \mathrm{e}$ time constant $\lambda$ of a first order response is given by the time required for the instrument output to respond to $1-1 /$ e of an instantaneous change in the input signal. The weighting function used here was

$$
W T(t)= \begin{cases}0, & t>0 \\ \lambda^{-1} e^{t / \lambda}, & t \leq 0,\end{cases}
$$

with $\lambda=2$ seconds and $t=-10,-9, \ldots-1,0$. The values of $W T(t)$ are normalized:

$$
\sum_{t=-10}^{0} w T(t)=1.0
$$

Each sample is thus composed of a weighted average of the ten preceding values, smoothing the series.

After this time lag has been introduced, a least squares regression is performed to determine the linear trend of the series. Cloud fields often exhibit changes of the short wave properties on a scale of $10^{2}$ miles, as the clouds thin or thicken. While this trend does not affect the mean of the samples, it will appear as a large period oscillation of $\bar{x}_{t}$ about $\mu$. 
As will be shown in the next section, this increases the apparent a.d. This increase is spurious and should be removed. The regression yields a polynomial of the form $y=a x+c$, where $a$ is the trend. This is subtracted from each point of the time series:

$$
x_{i}=x_{i}-(a i+c)+\mu \text {. }
$$

One other statistic, the standard deviation $\sigma$, was computed for each time series:

$$
\sigma=\left[\frac{1}{n} \sum_{i=1}^{n}\left(\mu-x_{i}\right)^{2}\right]^{1 / 2}
$$

This value is a function of the relative reflectivities of the stored array, as well as the cloud segment box sizes. These parameters are changed to represent real conditions through the use of the coefficient of variation (or relative dispersion) $\tau=\sigma / \mu$. The value of $\tau$ for the simulated cloud field may be adjusted to approximate the real values measured in previous field experiments. 
III. RESULTS

In general, the simulation model results show several basic characteristics of irradiance measurements from the aircraft platform. The most important property noted is that the averaging distance is inversely proportional to the height $h$ of the sensor above or below the cloud surface. This is expected because the f.o.v. increases with z; this smoothes the signal through the integration of a larger number of areal elements. Also, the larger f.o.v. decreases the relative weight of each individual element. This reduces the extreme values.

The rate of decrease of the averaging distance, however, is not as large as expected. This is due to the large scale oscillations of the time series about the mean. These changes force the cumulative mean out of the error interval and they are not as easily smoothed as the high frequency variations. This indicates that the a.d. is influenced in two regimes by separate effects.

This two-regime characteristic may be demonstrated by observing the behavior of the cumulative mean of a sinusoidal time series. Let the time series be defined as:

$$
y(t)=S \sin (\pi / 2 b t)+M,
$$

where $S=$ amplitude, $b=$ frequency $/ 4, t=$ time, $M=$ some mean bias value. As the cumulative mean $\bar{y}_{t}$ oscillates about $M$ it will reach peak values when the product $b t=1,5,9, \ldots$. The sum of the values in the series from $t=0$ to the first maximum is

$$
B=\sum_{t=0}^{1 / b} y(t)=\int_{0}^{1 / b} s \sin (\pi / 2 b t) d t+M / b .
$$

The point $t_{f}$ in the time series where $\bar{y}_{t}$ last exceeds the error interval $M \pm e M$ is given approximately by 


$$
t_{f} \cong \frac{B-M / b}{e M} \text {. }
$$

As an example, let $M=4$, and let $S=4, b=.1$ for small scale oscillations and $S=.5, b=1 / 50$ for the large scale features.

Substituting these values into Eq. (20) yields $B=\frac{80}{\pi}+40$ and $B=\frac{50}{\pi}+500$ for the small and large scales respectively. From Eq. (21), this would require a sample size of about 640 for the small scale effects and 400 for the large scale. This might represent the case of an aircraft flying near cloud base or cloud top. Close to the cloud surface the a.d. is the result of the high frequency oscillations. Farther away, however, these fluctuations diminish and the dominant effect becomes the large scale low frequency oscillations. Thus, the ultimate constraint on the a.d. is the large scale change in the mean value.

Further evidence of this may be seen in Table II which is a comparison of the averaging distance and the standard deviation for a number of simulations over non-uniform cloud decks.

Table II. Comparison of Averaging Distance and Standard Deviation.

\begin{tabular}{cccc}
$\mu$ & $\sigma$ & $\tau$ & $\begin{array}{c}\text { Averaging Distance } \\
\text { (\# areal elements) }\end{array}$ \\
\hline 3.532 & .503 & .142 & 2000 \\
3.476 & .492 & .142 & 1200 \\
3.404 & .456 & .134 & 2000 \\
3.516 & .426 & .121 & 1650 \\
3.437 & .481 & .140 & 1200 \\
\hline
\end{tabular}

$\sigma$ is primarily influenced by the large amplitude high frequency variations, but in this case, $\sigma$ does not seem to have any effect on the a.d. 
This indicates the large scale variations are the dominant effect here. The larger high frequency oscillations are superimposed on smaller oscillations of much longer wavelength as seen in Figure 5.

The a.d. is also affected by the cloud population. As the percent cloudiness varies, there are two factors which may change the a.d.: the size of the error interval, and the large scale cloud distribution. The error interval $\mu \pm \mathrm{e} \mu$ decreases linearly with the mean, causing the a.d. to increase. Perturbations about $\mu$ will force the cumulative mean outside a small error interval more readily than outside a larger one. Thus, for the above-cloud case, this means that the a.d. increases as the cloud cover decreases. The opposite effect is observed when sampling the downward irradiance from beneath the cloud deck.

The effect of the cloud population on large-scale variations, however, must also be taken into account. These variations of the cloudiness are a result of a local concentration of clouds or of clear areas. With a small (large) percent areal cloud cover, the clouds (clear areas) are widely spaced, so any relative concentrations of these areas will be small. A cloud cover of $50 \%$ presents the best opportunity for the grouping of a greater number of cloud or clear areas. Thus, the amplitude of the long period oscillations are at a maximum in this case.

To test this hypothesis, sample time series were simliated for $10 \%$, $50 \%$, and $90 \%$ cloud cover. These time series were then smoothed by taking 50-sample averages to reduce the high frequency variations. A power spectrum analysis was performed on each of the smoothed series to determine the contribution to the variance by the different scales of variation. This information was then plotted as spectral density times the frequency vs, the logarithm of the period. This representation was 


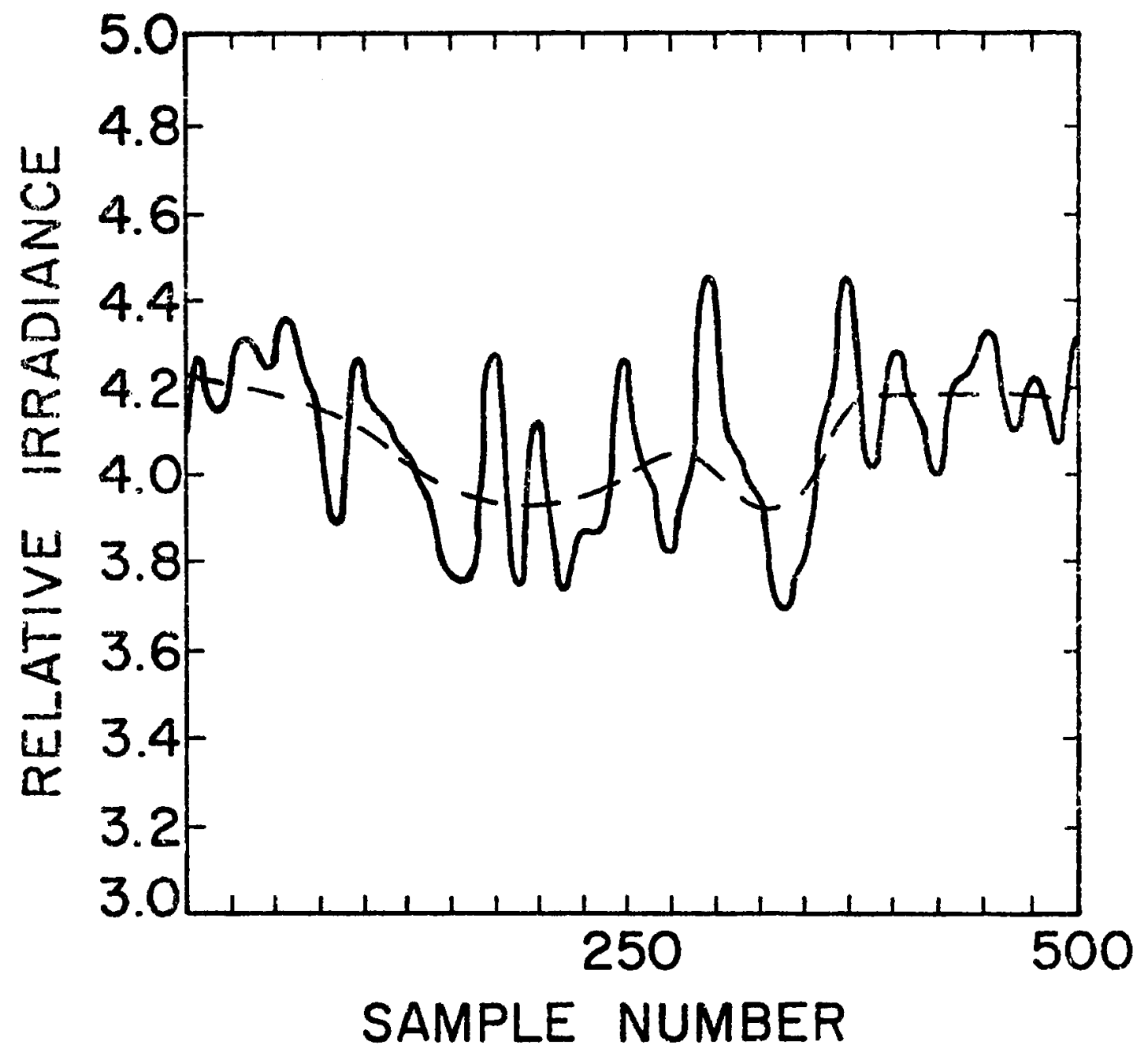

Figure 6. High frequency variations (solid line) superimposed on low frequency variations (dashed 7ine). Dashed line represents 50 sample averages. 
used because it has the advantage of being area conservative (Rasmussen and Balick, 1972). The results for five different randomly generated cloud fields are shown in Figure 7. As expected, the case of $50 \%$ cloud cover shows a significantly larger amount of variance in the low frequency end of the spectrum. Thus, in the regime where the low frequency variations are dominant, the largest averaging distances are found with $50 \%$ cloud cover. For example, the a.d. for $40 \%$ is greater than the a.d. for $20 \%$, and the a.d. for $60 \%$ is greater than the a.d. for $80 \%$.

One of several problems encountered in the development and operation of the model concerned the method of data analysis. The technique chosen could be termed a "brute force" method since it requires a large data set as it observes the course of the signal variations, and it does not employ any refined statistical techniques. Unfortunately, this choice was unavoidable because of the nature of the data. Most statistical methods of analysis assume the data samples to be independently and essentially normally distributed. The overlap of the f.o.v. for successive samples makes this irradiance data highly dependent. One measure of the dependency is the serial correlation coefficient $r *$ (Box and Jenkins, 1970):

$$
r * \cong \frac{\sum_{i=2}^{n} x_{i-1} x_{i}-\frac{\left(\sum_{i-1}^{n-1} x_{i}\right)\left(\sum_{i=2}^{n} x_{i}\right)}{n}}{(n-1) s^{2}}
$$

where $s^{2}$ is the sample variance. For all but the most uniform of conditions studies, $r^{*}$ was greater than .90 , indicating that the assumption of independency is not valid here. This point is emphasized by the fact that a simple correlation between the standard deviation and the a.d. may not be made, as shown earlier in Table II. 


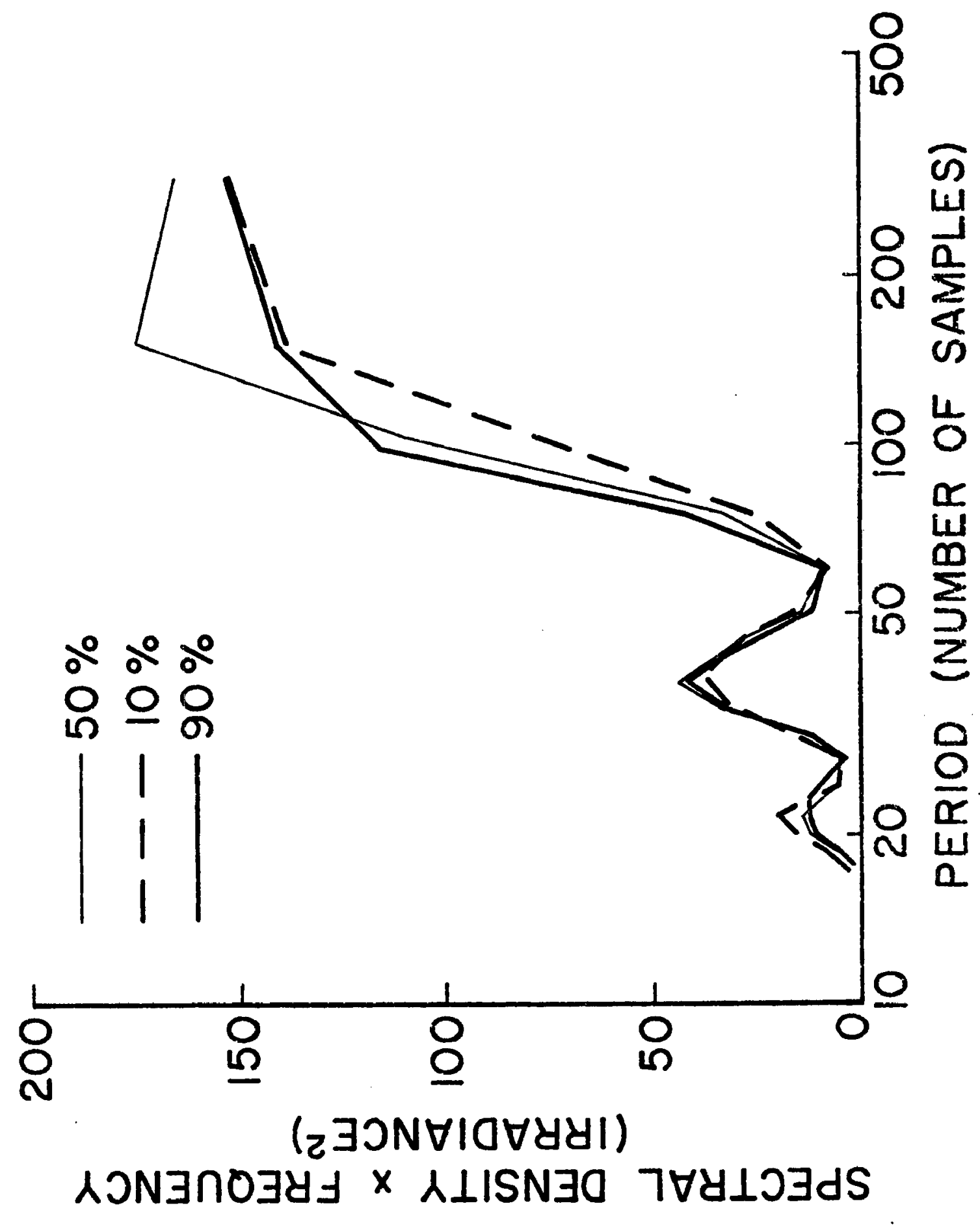

Figure 7. Power spectrum analys is of large scale variations in simulated irradiance time series, over cumulus cloud areal coverages of $10 \%, 50 \%, 90 \%$. 
Another problem which somewhat limited the operation of the model, is the large computer core space required for many of the runs. Attempts were made to reduce this requirement through a change in resolution of the stored cloud field, and by repeating a shorter data set. Unfortunately, for the randomly stored cases, the resolution change produced a significantly larger a.d. This bias is attributed to the juxtaposition of the cloud segments as they are randomly stored. The use of smaller boxes apparently changes the resulting cloud distribution or spacing. The repetition of a small data set also failed because it introduces large scale oscillations which increase the a.d. 


\section{APPLICATIONS OF RESULTS FOR THE GATE}

The end product of the model simulation for the GATE is a series of graphs for the six cloud types. These graphs are found in Appendix I. The purpose of these graphs is to guide determination of the minimum averaging distance and aircraft altitude from a direct observation of the cloud field. Each graph related the distance $z$ of the aircraft from the cloud surface to the a.d. for each cloud type.

\section{A. Averaging Distances for Six Cloud Types}

Table III lists the ranges used for the various input parameters according to cloud type. As a partial explanation of this table, consider an aircraft sampling the upward short wave irradiance above a broken cloud deck. For the simulated randomly dispersed cloud deck, a resolution of 100 meters and $x, y$ box dimensions of 4-6 are equivalent to cloud variations on the order of $.5 \mathrm{~km}$. The element values simulate a cloud reflectivity four times that of the underlying surface and atmosphere. This cloud field was stored in an array 3000 elements long. If .8 of the total area beneath the aircraft is covered by cloud and if the plane is $600 \mathrm{~m}$ above the cloud surface, this would require a sampling distance of $210 \mathrm{~km}$ for an accurate mean value. At an air speed of $100 \mathrm{~m} / \mathrm{sec}$, this would be a sampling run of 35 minutes. Similar logic applies to other applications of the tabular values. Appendix II contains a sample microfilm plot of each cloud type simulation except the uniform deck, for which no plots were made. 
Table IIIa. Averaging Distances for Simulated Randomly Dispersed Cloud Elements and Resulting Cloud Fields

\begin{tabular}{|c|c|c|c|c|c|c|}
\hline CLOUD TYPE & $\begin{array}{l}x, y \\
\text { BOX DIMENSIONS }\end{array}$ & $\begin{array}{l}\text { ELEMENT } \\
\text { VALUES }\end{array}$ & $\begin{array}{l}\text { ARRAY } \\
\text { LENGTH }\end{array}$ & $\begin{array}{l}\text { CLOUD } \\
\text { COVER }\end{array}$ & HEIGHT $z$ & $\begin{array}{l}\text { AVERAGING DISTANCE } \\
\text { (No. of El ements) }\end{array}$ \\
\hline Scattered Cumulus & $1-3$ & $\begin{array}{l}\text { Clear } 1 \\
\text { Cloud } 4\end{array}$ & 3000 & $\begin{array}{l}.10 \\
.20 \\
.40\end{array}$ & $\begin{array}{r}3 \\
6 \\
10 \\
3 \\
6 \\
10 \\
3 \\
6 \\
10\end{array}$ & $\begin{array}{r}2250 \\
1400 \\
650 \\
2250 \\
1400 \\
650 \\
2350 \\
1900 \\
1500\end{array}$ \\
\hline Broken Deck & $4-6$ & $\begin{array}{l}\text { Clear } 1 \\
\text { cloud } 4\end{array}$ & 3000 & $\begin{array}{l}.60 \\
.80\end{array}$ & $\begin{array}{l}3 \\
6 \\
9 \\
3 \\
6 \\
9\end{array}$ & $\begin{array}{r}r 3000 \\
2700 \\
2400 \\
2300 \\
2100 \\
1850\end{array}$ \\
\hline Uniform Deck & $10-14$ & $37-43$ & 1000 & 1.00 & $\begin{array}{r}3 \\
6 \\
10\end{array}$ & $\begin{array}{r}270 \\
105 \\
80\end{array}$ \\
\hline Stratocumulus & $80-120$ & $\begin{array}{l}\text { Clear } 1 \\
\text { Cloud } 4\end{array}$ & 3000 & 2.80 & $\begin{array}{l}3 \\
6 \\
9\end{array}$ & $\begin{array}{r} \\
3000 \\
2800 \\
2600\end{array}$ \\
\hline
\end{tabular}


Table IIIb. Averaging Distances for

Fixed Coordinate Cloud Fields

\begin{tabular}{|c|c|c|c|c|c|c|}
\hline CLOUD TYPE & $\begin{array}{l}x, y \\
\text { BOX DIMENSIONS }\end{array}$ & $\begin{array}{l}\text { ELEMENT } \\
\text { VALUES }\end{array}$ & $\begin{array}{l}\text { ARRAY } \\
\text { LENGTH }\end{array}$ & $\begin{array}{l}\text { CLOUD } \\
\text { COVER }\end{array}$ & HEIGHT $z$ & $\begin{array}{l}\text { AVERAGING DISTANCE } \\
\text { (No. of El ements) }\end{array}$ \\
\hline Street Cumulus & $6 \times 7$ & $\begin{array}{l}\text { Clear } 1 \\
\text { Cloud } 5\end{array}$ & 250 & .07 & $\begin{array}{r}3 \\
6 \\
9 \\
15\end{array}$ & $\begin{array}{r}1000 \\
500 \\
250 \\
110\end{array}$ \\
\hline Band Clouds & $\begin{array}{l}\text { Band Widths } \\
2 \text { thru } 25\end{array}$ & $\begin{array}{l}\text { Clear } 1 \\
\text { Cloud } 6\end{array}$ & 250 & $\begin{array}{l}.33 \\
.50\end{array}$ & $\begin{array}{r}1.5 \\
2 \\
3 \\
4.3 \\
6 \\
10 \\
15 \\
1.5 \\
2 \\
3 \\
3.75 \\
6 \\
10 \\
15\end{array}$ & $\begin{array}{r}2000 \\
1400 \\
800 \\
450 \\
250 \\
75 \\
20 \\
1000 \\
700 \\
370 \\
250 \\
85 \\
15 \\
2\end{array}$ \\
\hline
\end{tabular}


Some adjustments were made in the random storing technique as it was applied to cloud types other than a broken cloud deck. For scattered cumulus, the cloud boxes were stored as squares. The sizes were weighted to produce a distribution of predominantly small cumulus similar to that reported by Planck (1969). The essential feature of the distribution that was reproduced in this simulation is the logarithmic decrease of the cloud density with cloud diameter, shown in figure 8.

The uniform overcast deck utilized six bits per stored value instead of three. This allowed a range of double digit integer values to give more resolution over a small range. The doubling of the size of the stored array was permissible in this case because the uniform deck has a relatively short a.d. The values were centrally weighted about the mean to enhance the uniformity of the stored array.

The stratocumulus clouds were approximated by large rectangles stored with clear strips on all sides to prevent overlap. While this shape may not appear realistic, it does offer the opportunity to study a cloud field with large scale variations.

A special analysis was made in the case of street cumulus. The distribution and spacing of the simulated clouds was estimated from the tropical sky study of Malkus and Rieh1 (1964). The present analysis of this type of cloud distribution was centered on determining a minimum separation of the aircraft and the clouds as well as an optimal a.d. In order to derive a precise mean of the irradiance field, the effects of both the altitude and the orientation of the flight track relative to the clouds must be considered. It was determined here that the sampling runs should be made perpendicular to the cloud lines at a minimum relative height $z=9$ units from the cloud surface. A cloud-sensor 


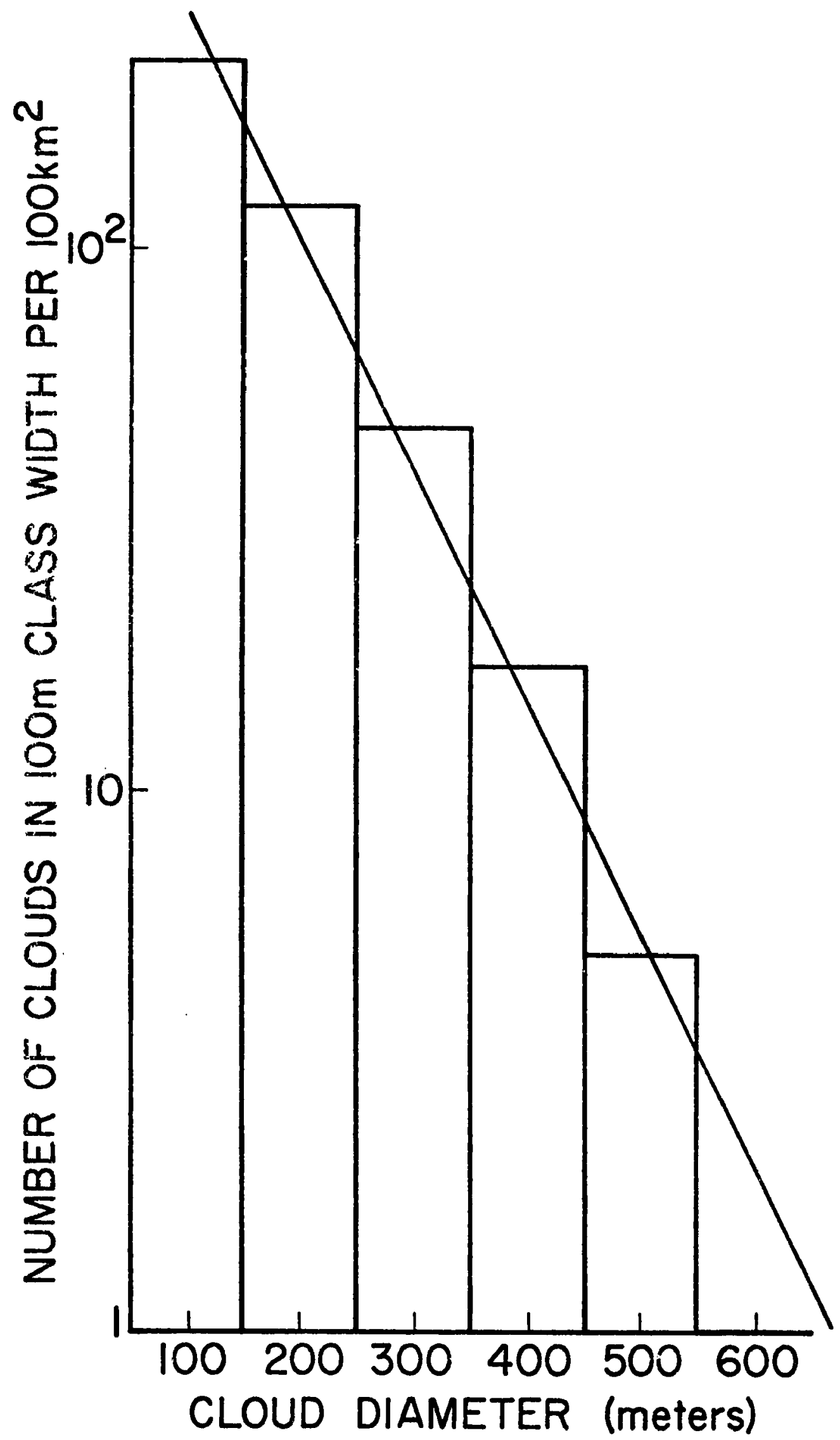

Figure 8. Simulated distribution of cumulus clouds. 
separation of less than this is likely to result in an erroneous biased mean value depending on whether the sensor passes over the clouds or over the clear spaces between.

\section{B. A Bi-Modal Analysis Technique}

A slightly different technique of data analysis from that developed earlier in this paper may be applied to large-scale cloud features. This method would be employed in cases where the irradiance at the sensor exhibits a bi-modal distribution of maximum and minimum values from cloud and clear areas. In this case, the mean irradiance would be calculated as an area weighted mean of the clear and cloud reflected radiance values.

$$
\bar{x}=\frac{{ }_{C L R}{ }^{x} C L R+A_{C L D} x_{C L D}}{{ }_{C L R}+A_{C L D}},
$$

where $A_{C L R}$ and $A_{C L D}$ are the total amounts of clear and cloud areas;

${ }^{x}$ CLR and $x_{C L D}$ are the mean irradiance values for those samples where the f.o.v. of the sensor is filled by a clear or cloud area respectively. The areal coverages are to be determined by a threshold value $x_{\mathrm{TV}}:{ }_{\mathrm{CLR}}$ $\left(A_{C L D}\right)$ is the number of samples $x_{j}$ such that $x_{j}<x_{T V}\left(x_{i}>x_{T V}\right) . x_{T V}$ is the numerical average of $x_{C L R}$ and $x_{C L D}$.

This method was tested by simulating an irradiance time series with a one-dimensional array of random integers. If all odd numbers (clear areas) are replaced with a value of one and all even numbers (representing cloud) are assigned a value of four, this represents a simulated time series of relative reflected irradiances. This series is smoothed by taking a running average over several values. The size of the cloud and clear areas may be varied by replacing each random value with a number of the same relative reflected irradiances. For example, $x=2,6,6,7, \ldots$ may be replaced by $x=4444,4444,4444,1111, \ldots$ 
The applicability of this scheme to a given set of irradiance values is indicated by the coefficient of variation $\tau$ of the samp?e values. This statistic shows the extent to which the series distribution is bi-modal. Recalling that $\tau=\sigma / \mu, \tau$ increases as the distribution becomes more bi-modal. Preliminary results set the lower limit of $\tau$ for the use of this technique at $\tau \sim .5$.

The accuracy of this method is affected by the choice of a threshold value and by the length of the sample time series. By varying the threshold value $x^{\prime}$ TV for many series of random integers it was found that $x^{\prime}{ }_{T V}$ may range within the approximate limits of $x_{T V} \pm(.15) x_{T V}$. The variations did not signficantly affect the mean $\bar{x}$ as calculated from Eq. (23). Larger or smaller values of $x^{\prime}{ }_{T V}$ may distort the areal weighting. A series length $n$, such that $\sigma / n>.01$, was found to contain sufficient data for an accurate calculation of $\bar{x}$. These lengths were found to be much less than those shown in Table IIIa for a similar case of closed cellular convection.

This brief investigation has produced only some approximate limits to the validity of the bi-modal technique presented above. However, it has indicated a possible method for the analysis of large scale cloud features that does not require an extensive amount of data.

\section{Applications to Surface Measurements of Irradiance}

The results of this irradiance measurement simulation could also be applied to the time series of surface observations. The motion of the sensor relative to the clouds would be the speed of cloud propogation by the mean wind. Since this is roughly an order or magnitude slower than aircraft speeds simulated here, this implies sampling times on the order of hours, particularly for low level clouds. The assumption of intransient 
atmospheric conditions does not hold on this scale, and the path length of the sun's rays through the atmosphere would change significantly over this time. Thus, for heterogeneous partly cloudy conditions it does not seem feasible to precisely determine the mean short wave irradiance from a stationary platform at the surface, particularly for use in calculating instantaneous heating rates. Mean values as measured from a surface platform are also not likely to be representative of a large area. 


\section{CONCLUSIONS}

A computer simulation has been developed to optimize the use of the aircraft platform for the GATE Radiation Subprogram. As part of this subprogram, radiative divergence profiles of the tropical atmosphere will be derived from aircraft sampling of mean radiative fluxes. Optimal use of allocated aircraft time depended on the acquisition of sufficient data for calculating accurate mean values without oversampling or undersampling. This model was used to simulate the measurement of radiative fluxes to determine the approximate sample sizes required under various conditions of cloudiness.

The sample size or averaging distance was found, in general, to be inversely proportional to the height of the sensor above or below the cloud field. This is due to a smoothing of the signal by the instrument as the diameter of the projection of its field of view increases with height. The magnitude of the averaging distance and the rate of its decrease with height is the result of signal variations on two spatial scales. Near the cloud surface, small scale large amplitude changes in irradiance give the data a large sample variance. This must be reduced by increasing the sample size. As the height difference between the aircraft and the cloud surface increases, these high frequencies are rapidly filtered by the instrument. Further from the cloud, large scale changes in the cloud properties become the dominant effect. These oscillations are not as easily smoothed and the averaging distance does not decrease as quickly with height. The large scale effects are thus the overriding control on the averaging distance.

For the GATE specific cloud types were analyzed and averaging distances computed. These results were compiled into a series of graphs 
to permit a real time decision on aircraft sampling altitudes and distances, and to assist in the subsequent analysis of the data. Only a narrow range of conditions were sampled for this experiment since it is not feasible to cover all possibilities and resources were limited. The cases selected here are representative and should provide a much needed set of guidelines. 
REFERENCES

Box, G. E. P. and G. M. Jenkins, 1970: Time Series Analysis. Holden-Day, San Francisco, 553 pages.

Cox, S. K., 1973: Radiation Components of the Energy Budget for BOMEX. Atmospheric Science Paper No. 208, Dept. of Atmospheric Science, Colorado State University, Fort Collins, CO 80523, 43 pages.

Cox, S. K. and T. H. Vonder Haar, 1973: Measurements of Solar Energy for the Study of Time Dependent Meteorological Systems. Presented at the Symposium on Solar Radiation, Smithsonian Institution, Rockville, Maryland.

Kraus, H., et a1, 1973: The Radiation Subprogram for the GARP Atlantic Tropical Experiment. GATE Report No. 4, International Council of Scientific Unions, World Meteorological Organization, 109 pages.

Malkus, J. S. and H. Rieh1, 1964: Cloud Structure and Distributions Over the Tropical Pacific Ocean. University of California Press, Berkeley and Los Angeles, 229 pages.

Planck, V. G., 1969: The Size Distribution of Cumulus Clouds in Representative Florida Populations. J. Appl. Meteor., 8, pp. 46-67.

Rasmussen, J. L. and L. K. Balick, 1972: A Study of the Cloud Top Temperature of an Orographic Cloud. SNOPSET Final Report, Dept. of Mechanical Engineering, Colorado State University, Fort Collins, Colorado, 102 pages.

Reed, R. J. and E. E. Recker, 1971: Structure and Properties of SynopticScale Wave Disturbances in the Equatorial Western Pacific. J. Atmos. Sci., 28, pp. 1117-1733.

Salomonson, V. V. and W. E. Marlatt, 1968: Anisotropic Solar Reflectance Over White Sand, Snow, and Stratus Clouds. Atmospheric Science Paper No. 120, Colorado State University, Fort Collins, CO 80523 41 pages.

Sikula, G. J. and T. H. Vonder Haar, 1972: Very Short Range Local Area Weather Forecasting Using Measurements from Geosynchronous Meteorological Satellites. Atmospheric Science Paper No. 185, Colorado State University, $7 \overline{3}$ pages.

Suomi, V. E. and P. M. Kuhn, 1958: An Economical Net Radiometer. Te11us, 10, pp. 160-163.

Yanai, M., S. Esbensen, and J. H. Chu, 1973: Determination of Bulk Properties of Tropical Cloud Clusters from Large Scale Heat and Moisture Budgets. J.Atmos. Sci., 30, pp. 611-627. 


\section{APPENDIX A: Averaging Distances for the GATE Radiation Aircraft Program}

The following set of graphs presents the averaging distances calculated for the sampling of six cloud types in the GATE. These cases include scattered cumulus, broken stratiform cloud decks: uniform overcast cloud decks, stratocumulus in the form of large closed convective cells, organized "street" cumulus, and cloud bands. The ordinate of the graphs is the distance of the aircraft from cloud top or cloud base, and the abscissa is the distance that must be flown to obtain a significant mean value of the irradiance. Each curve on the graphs gives these two variables as a function of cloud cover for both above-cloud and sub-cloud sampling passes. These graphs were intended for real time use in the GATE and in the subsequent analysis of the GATE radiation aircraft data. 


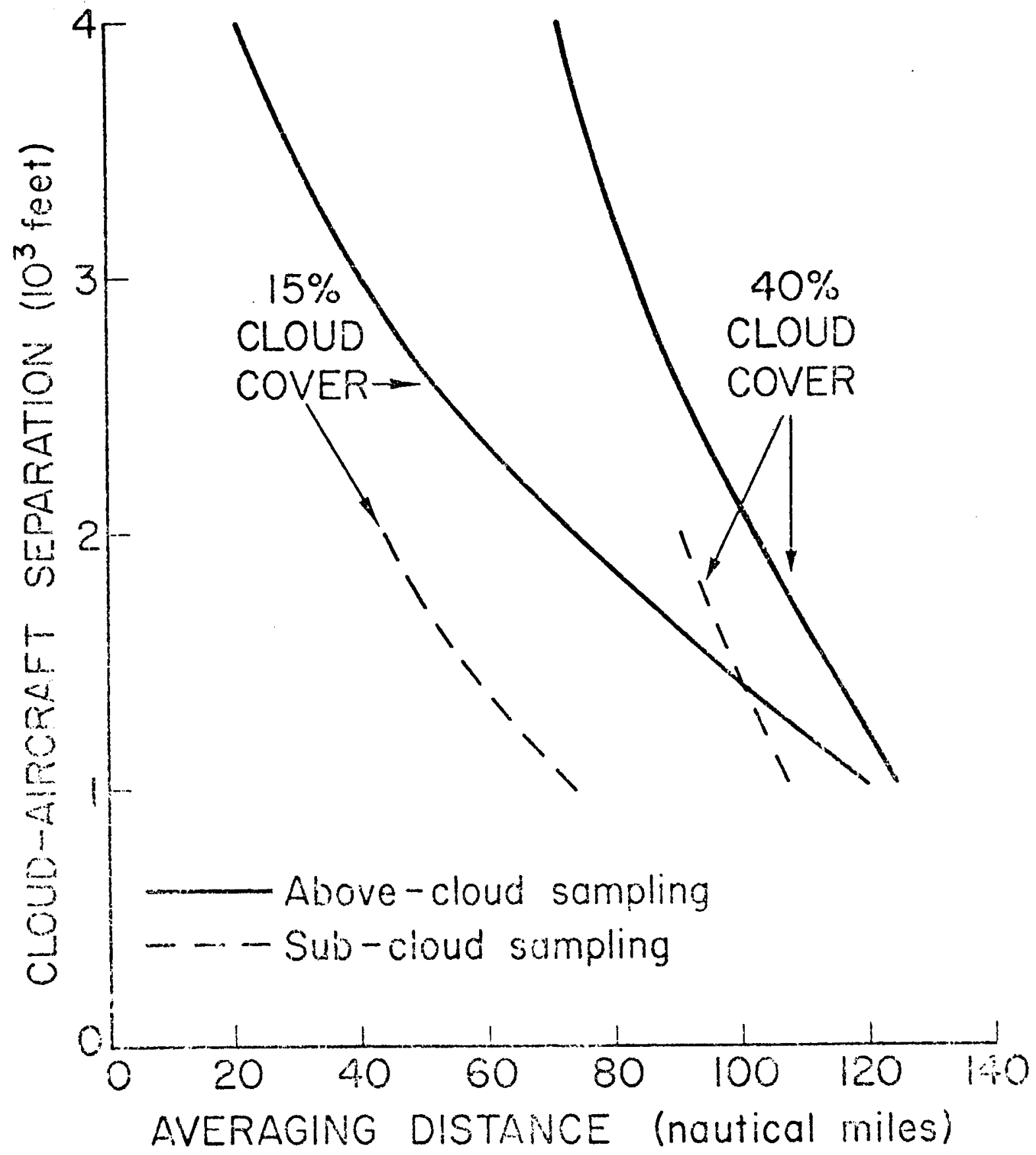

Figure A1. Averaging distance for scattered cumulus as a function of cloud-aircraft separation and \% cloud cover. 


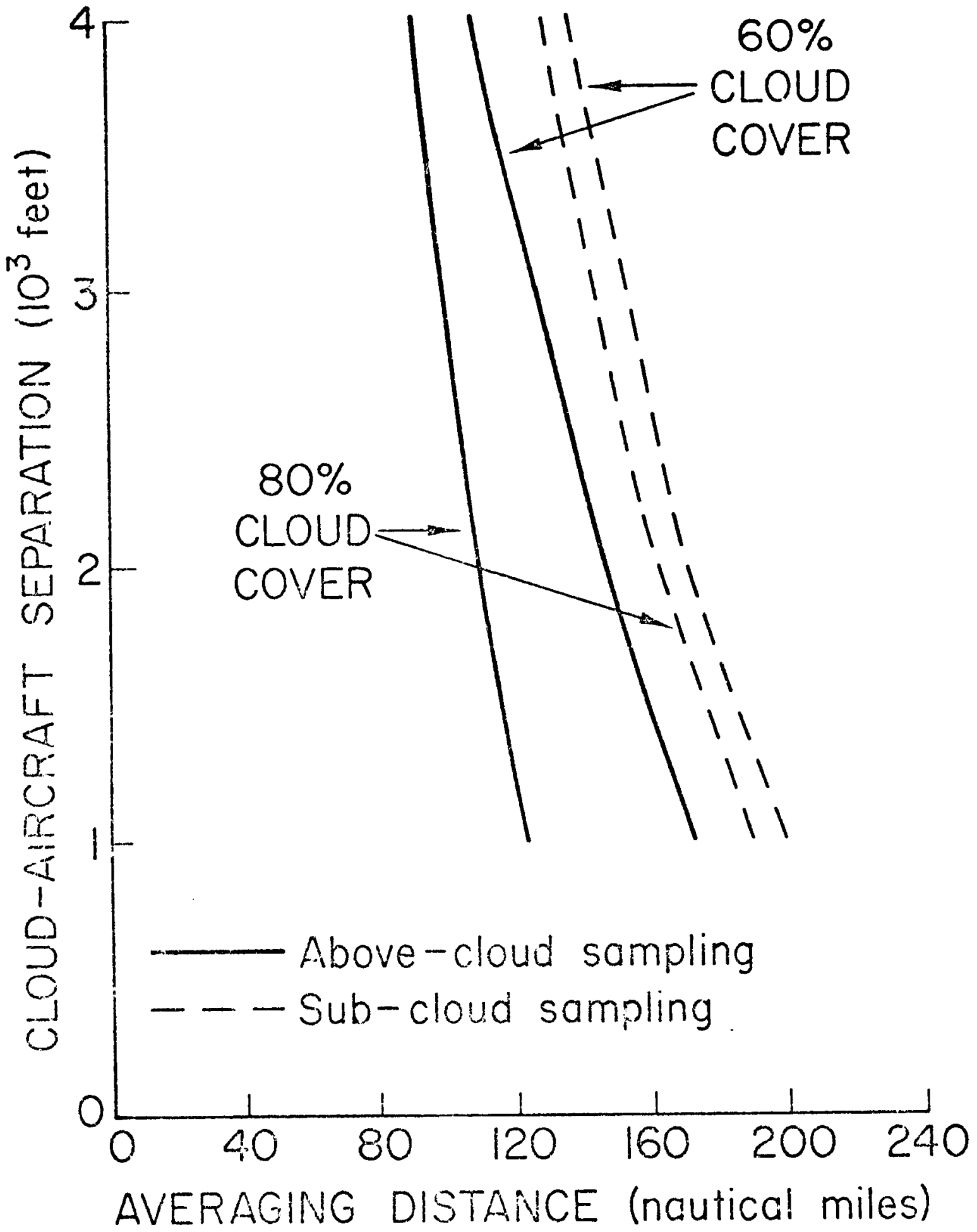

Figure A2. Same as Figure A1, but for broken cloud deck. 


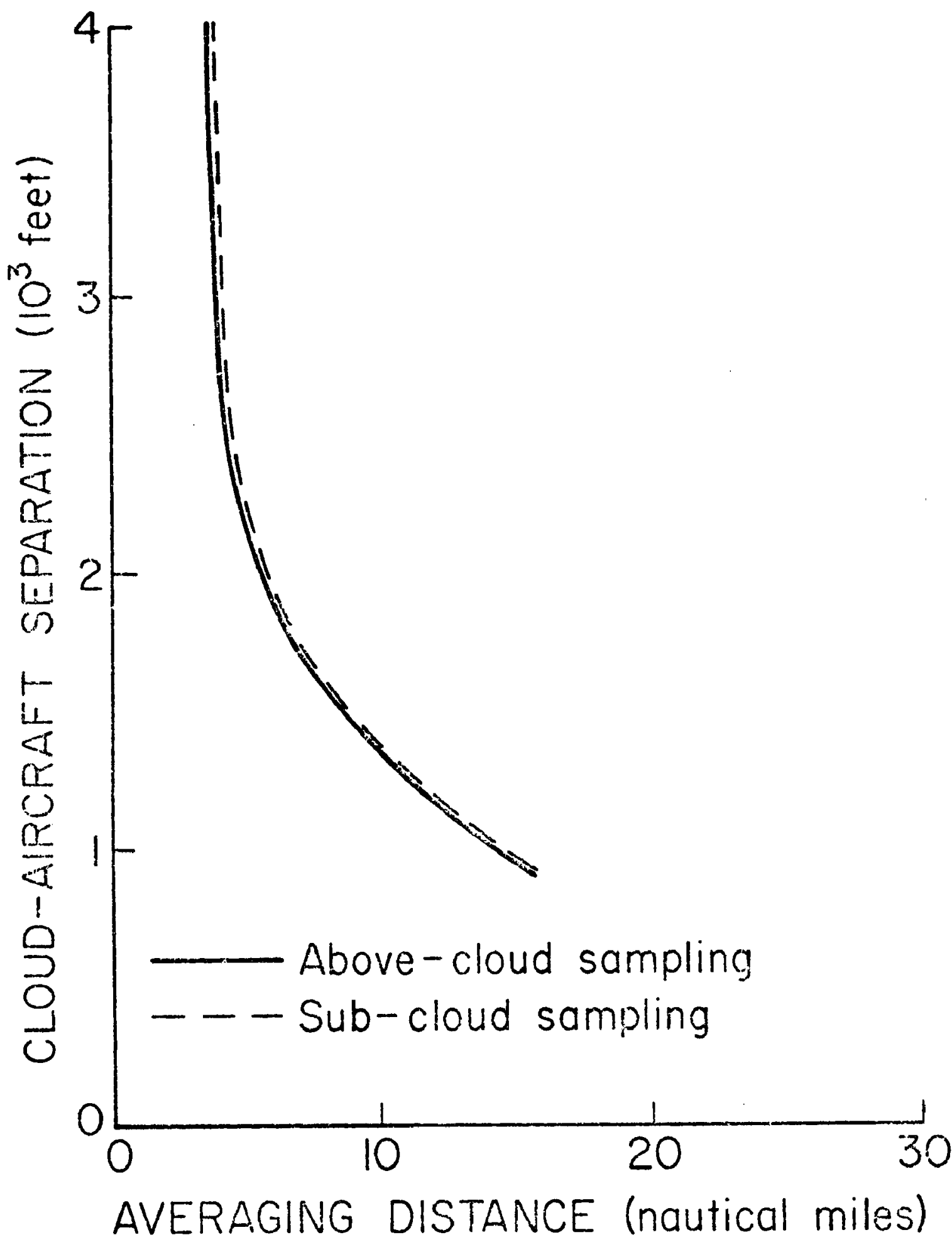

Figure A3. Averaging distance for uniform cloud deck as a function of cloud-aircraft separation. 


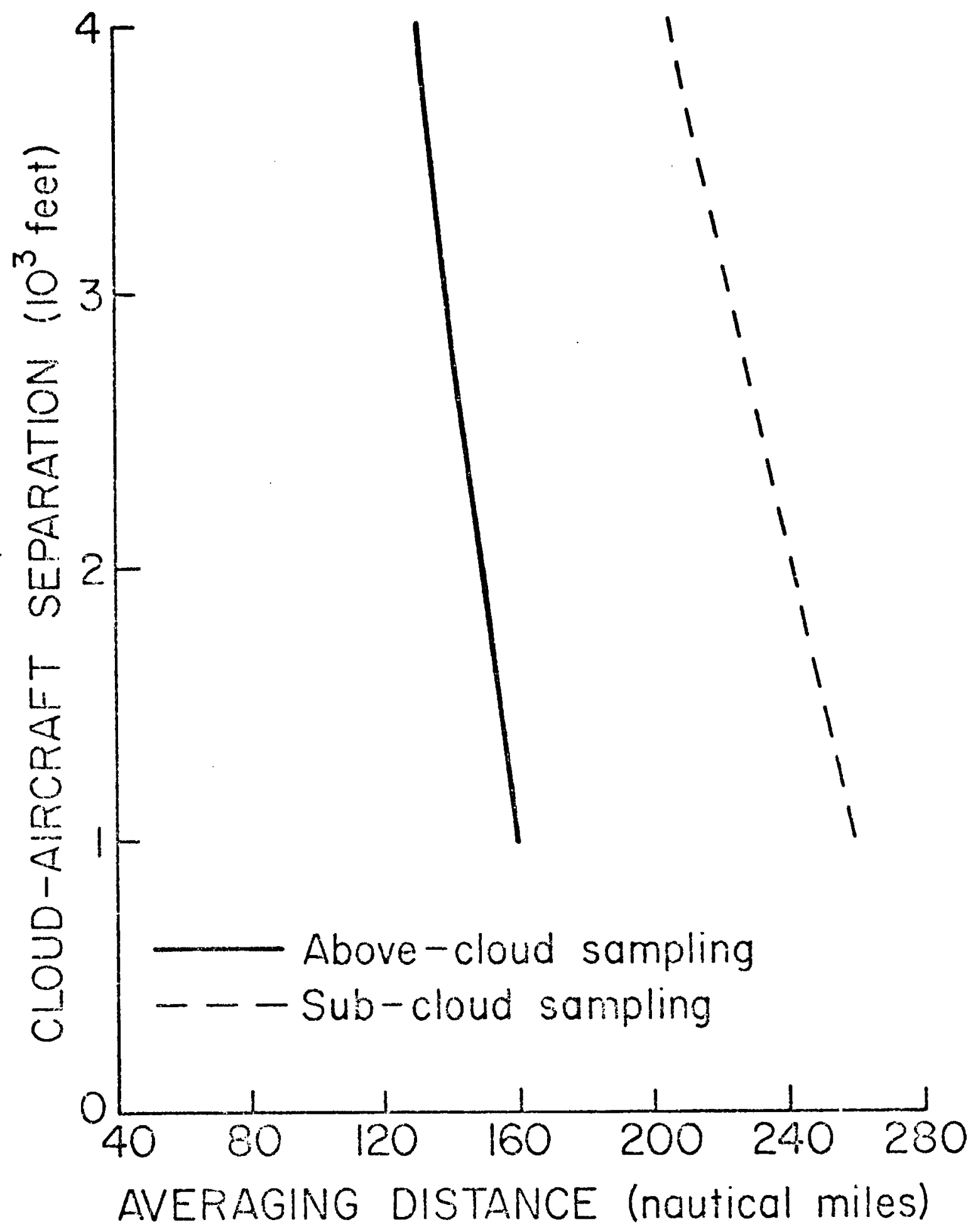

Figure A4. Same as Figure A3, but for closed cellular convection. 


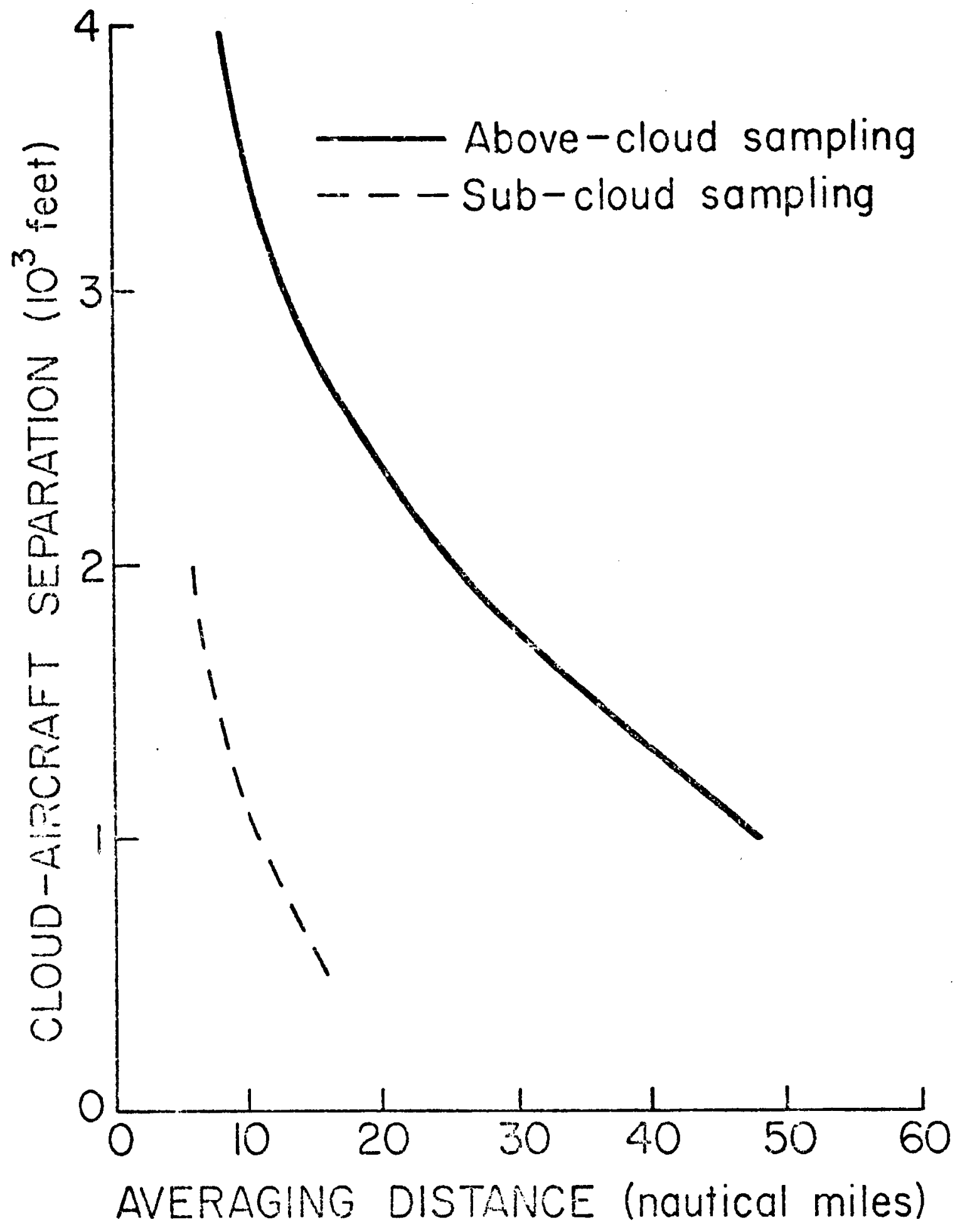

Figure A5. Same as Figure A3, but for street cumulus. 


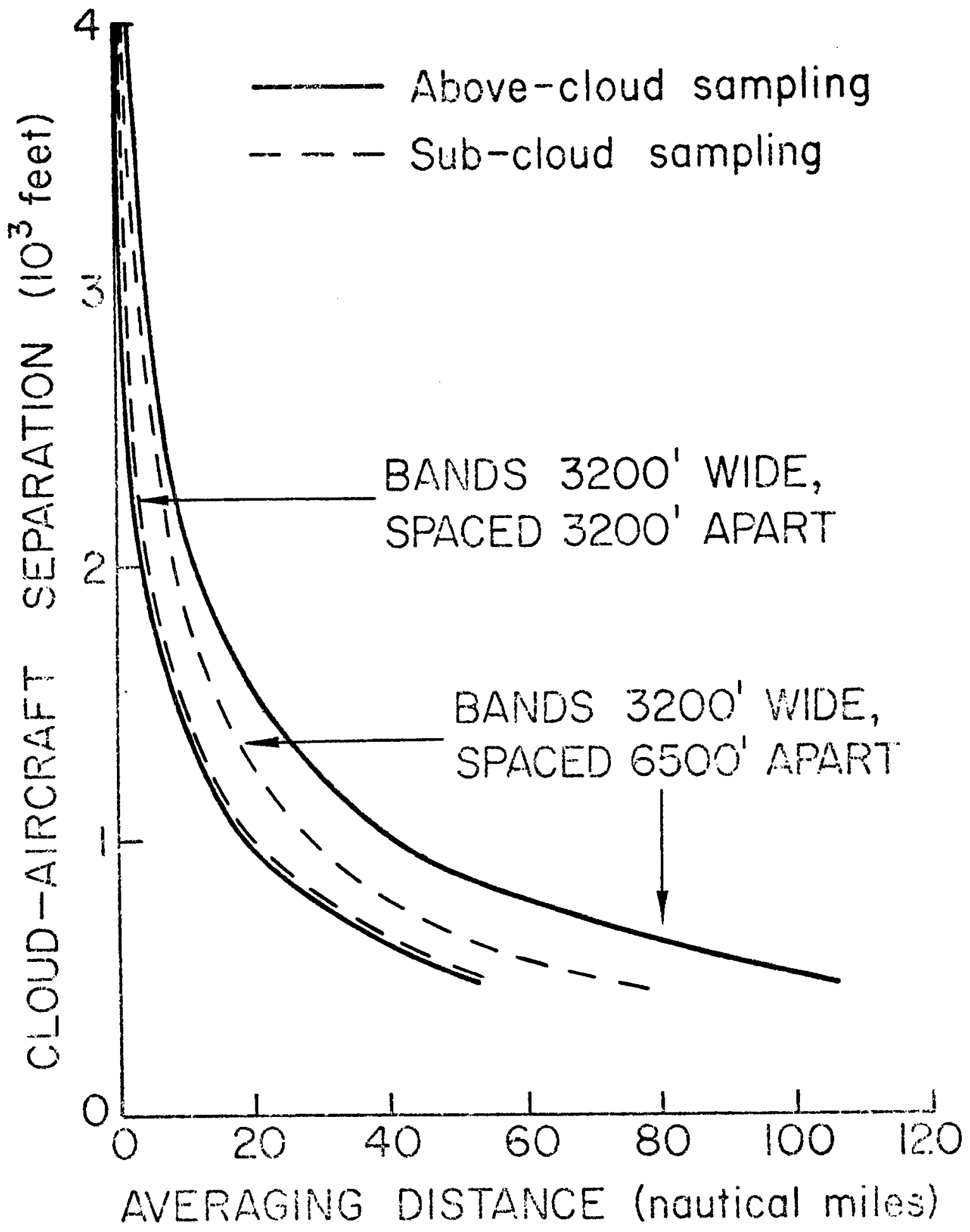

Figure $A 6$. Same as Figure $A 3$, but for cloud bands. 
APPENDIX B. Sample Simulated Cloud Arrays

Microfilm plots were generated for five of the six cloud types simulated in this study. The following figures are samples of the simulated cloud arrays as viewed from above. Cloud elements are represented by the white areas and clear regions by the dark areas. The resolution for the smallest cloud is 100 meters.

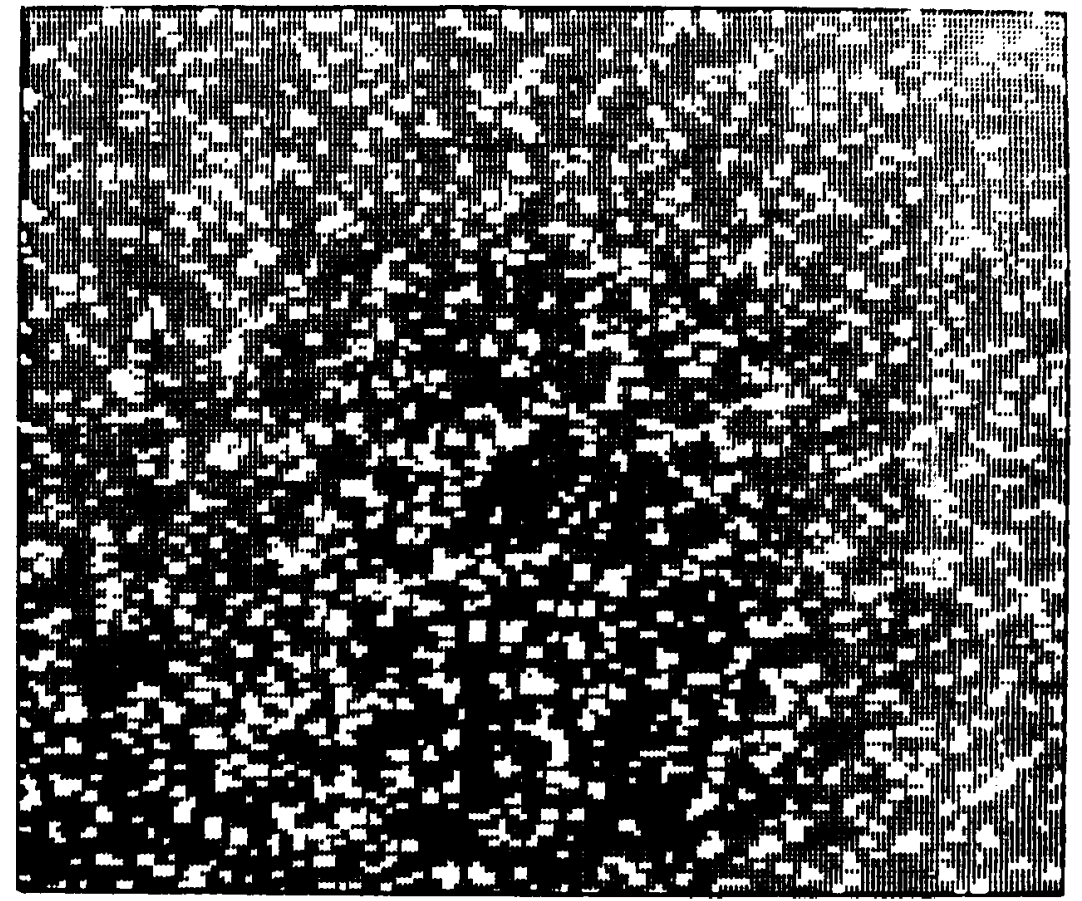

Figure B1. Plot of simulated cumulus cloud array, $20 \%$ areal coverage. 


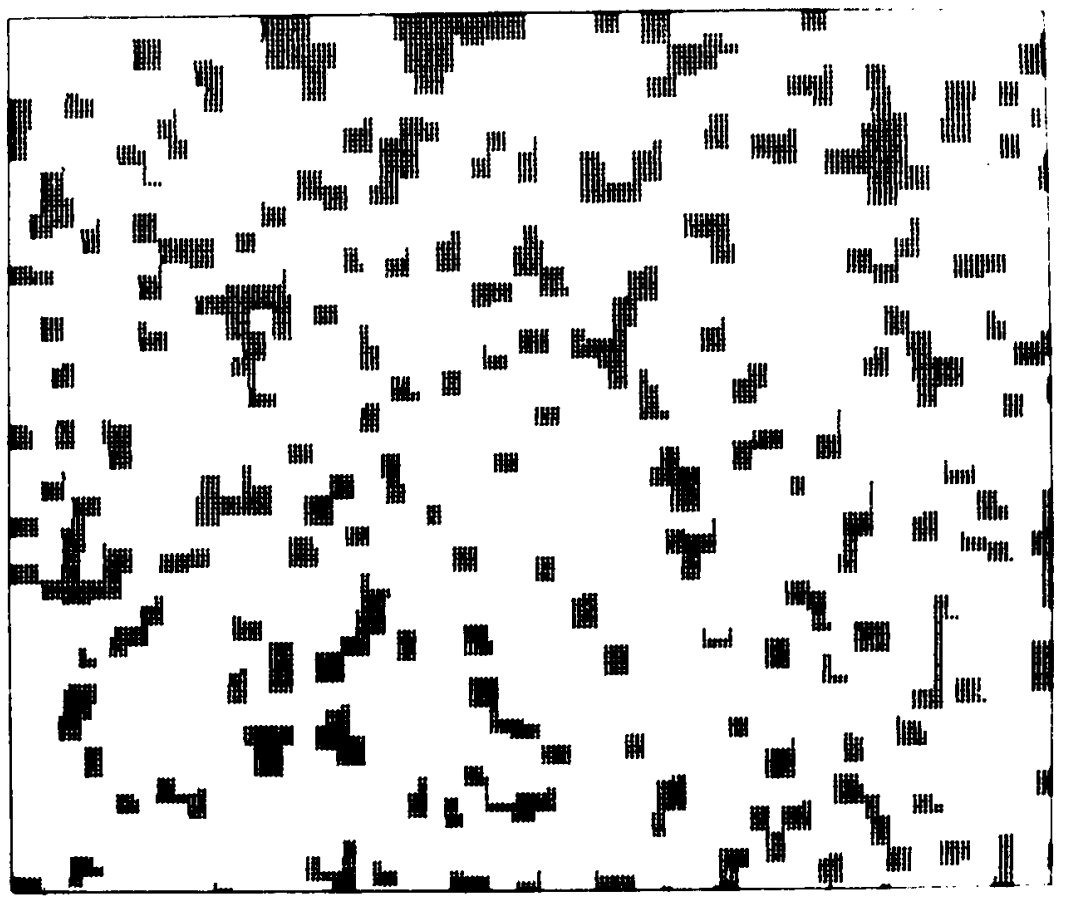

Figure B2. Plot of simulated broken cloud deck, $80 \%$ areal coverage.

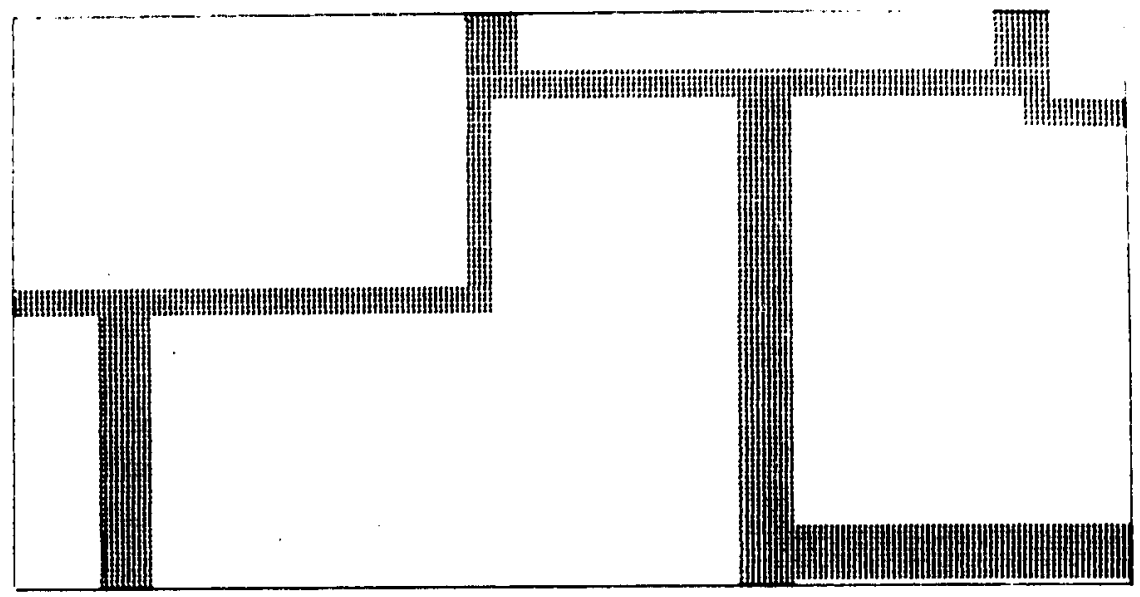

Figure B3. Plot of simulated closed cellular convection. 


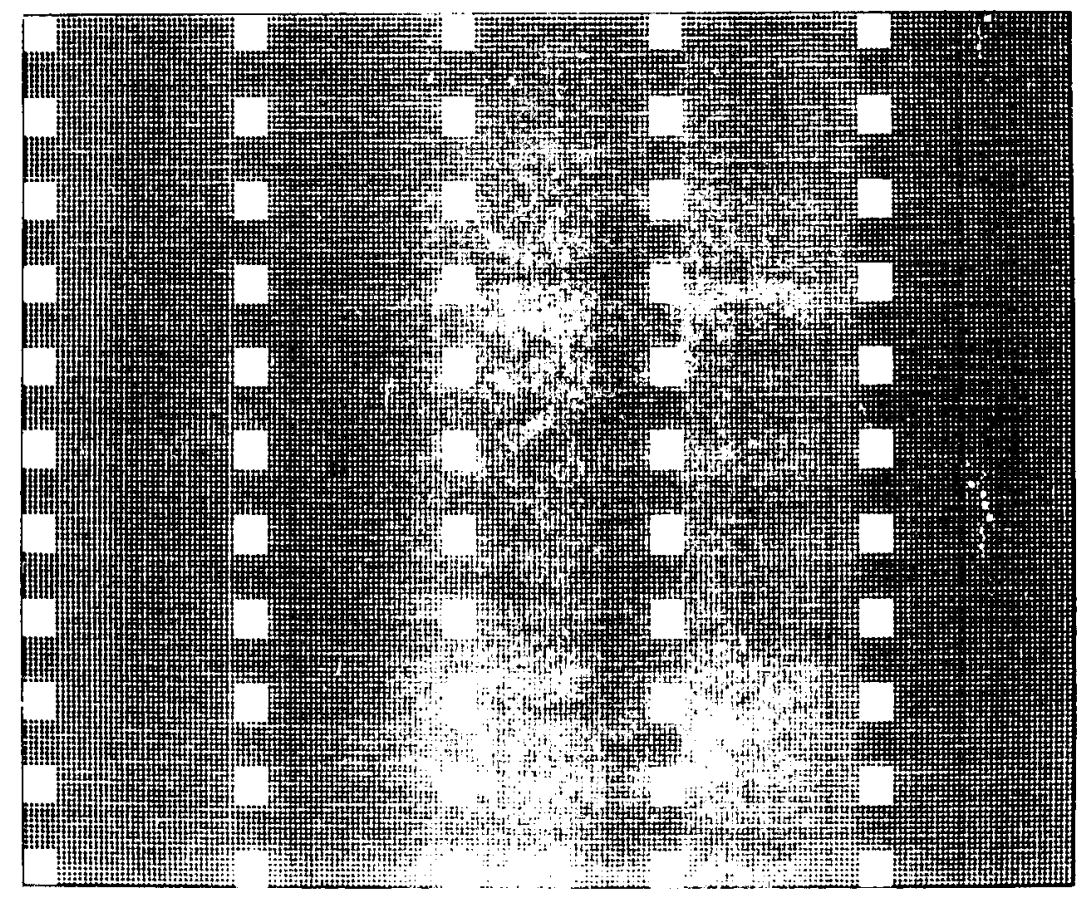

Figure B4. Plot of simulated street cumulus.

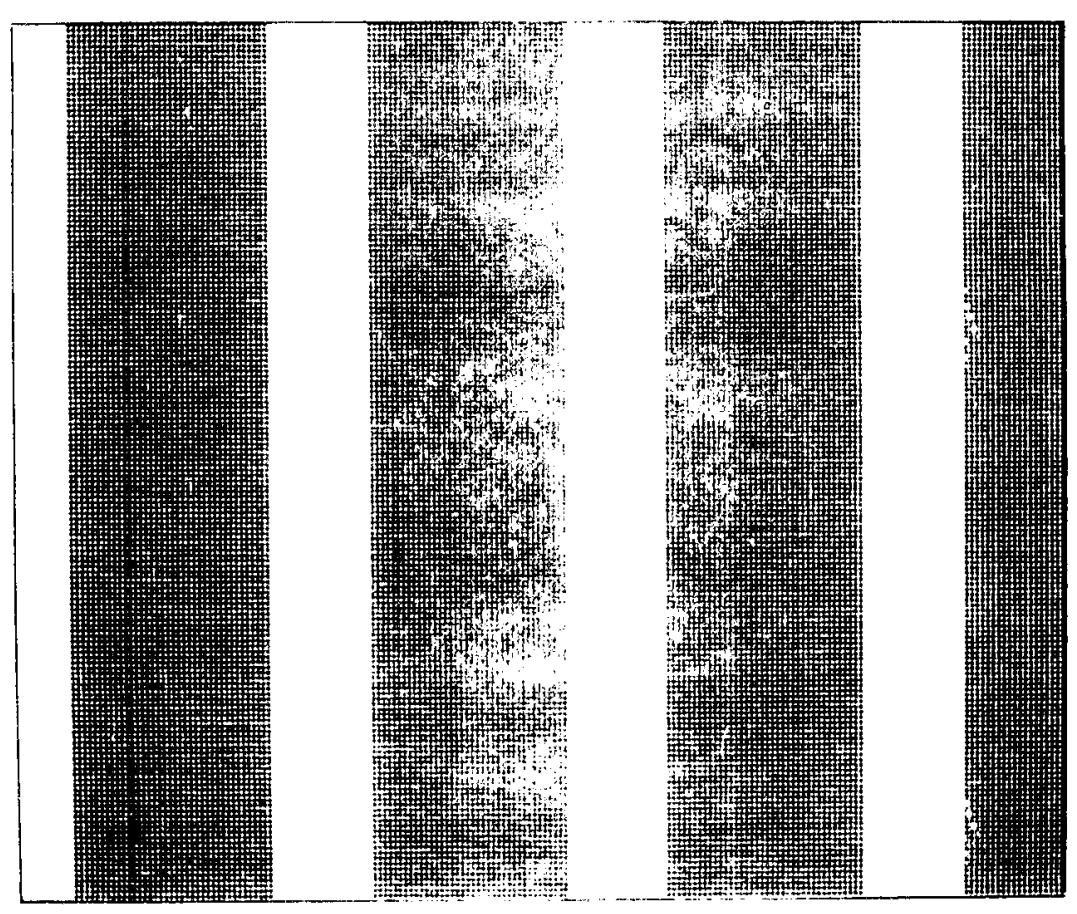

Figure B5. Plot of simulated cloud bands. 
BIBLIOGRAPHIC DATA SHEET

4. Title sni Jubticle

Computer Simulation of Irradiance Measurements from Aircraft

\begin{tabular}{|l}
\hline Computer Simulation of Irradiance Me \\
\hline 7. Aurhor(s) \\
Michael R. Poellot and Stephen \\
9. Performing Organizacion lame and Address \\
Department of Atmospheric Scic \\
Colorado State University \\
Fort Collins, Colorado 80523 \\
12. Sponsoring Organization Same and iddress \\
Atmospheric Sciences Section \\
National Science Foundation \\
Washington, D. C. 20550
\end{tabular}

15. Supplementary Sotes

16. Abscracts A computer simulation has been developed to optimize the use of the aircraft platform for the measurement of short wave irradiances. This model simulates the measurement of radiative fluxes in order to determine the approximate sample sizes required under various conditions of cloudiness.

The simulated required sampling length or averaging distance was found to be inversely proportional to the height of the sensor above or below the cloud field. The magnitude of the averaging distance and the rate of its decrease with height are the result of signal variations on two scales. Near the cloud surface, the data has a high variance due to small scale, large amplitude variations in the irradiance. These fluctuations are rapidly smoothed as the aircraft-cloud separation increases. The longer period oscillations are not as easily smoothed. When the aircraft is farther from the cloud, the large scale effects become the primary control on the averaging distance.

Six specific tropical cloud types were analyzed and averaging distances computed using this simulation.

17. Key Hords and Document Inalysis. 17a. Descriptors

Radiation Measurements

Airborne Radiation Measurements

17b. Identifiers/Open-Ended Terms

17e. Cost Tl Field/Group

\begin{tabular}{|c|c|c|c|}
\hline \multirow[t]{2}{*}{ 18. Avail ability statement } & & $\begin{array}{l}\text { 19. Securiey Class (This } \\
\text { Repore) } \\
\text { (ViCldSSIFIED }\end{array}$ & 21. To. of Pages \\
\hline & . & $\begin{array}{l}\text { 20. Securby Glaso (this } \\
\text { Page } \\
\text { WCLASIFIFD }\end{array}$ & 22. Pince \\
\hline
\end{tabular}

